\title{
Size and Connectivity as Emergent Properties of a Developing Immune Network
}

\author{
Rob J. De Boer and Alan S. Perelson \\ Theoretical Division, Los Alamos National Laboratory Mail Stop K710, \\ Los Alamos, NM 87545, U.S.A. \\ (Received on 6 April 1990, Accepted in revised form on \\ 12 September 1990)
}

The development of the immune repertoire during neonatal life involves a strong selection process among different clones. The immune system is genetically capable of producing a much more diverse set of lymphocyte receptors than are expressed in the actual repertoire. By means of a model we investigate the hypothesis that repertoire selection is carried out during early life by the immune network.

We develop a model network in which possibly hundreds of B cell clones proliferate and produce antibodies following stimulation. Stimulation is viewed as occurring through receptor crosslinking and is modeled via a log bell-shaped doseresponse function. Through secretion of free antibody $\mathbf{B}$ cell clones can stimulate one another if their receptors have complementary shapes. Receptor shapes are modeled as binary strings and complementarity is evaluated by a string matching algorithm. The dynamic behavior of our model is typically oscillatory and for some parameters chaotic. In the case of two complementary B cell clones, the chaotic attractor has a number of features in common with the Lorenz attractor. The networks we model do not have a predetermined size or topology. Rather, we model the bone marrow as a source which generates novel clones. These novel clones can either be incorporated into the network or remain isolated, mimicking the non-network portion of the immune system. Clones are removed from the network if they fail to expand. We investigate the properties of the network as a function of $P$ (match), the probability that two randomly selected immunoglobulins have complementary shapes.

As the model networks evolve they develop a number of self-regulatory features. Most importantly, networks attain a specific equilibrium size and generate a characteristic amount of "natural" antibody. Because the network reaches an asymptotic size even though the bone marrow keeps supplying novel clones, clones must compete for presence in the network, i.e. repertoire selection takes place. Networks comprised of cells with multireactive receptors remain small, whereas networks consisting of cells with specific receptors become much larger. We find an inverse relationship between $n$, the number of clones in a network, and $P$ (match), and a linear relationship between $n$ and $M$, the rate at which novel clones are produced in the bone marrow. We present a simple phenomenological model for the number of clones in the network that accounts for the inverse relationship between $n$ and $P$ (match), and that can account for the relationship between $n$ and $M$. Additionally, the phenomenological model suggests that there are two qualitatively different network equilibria.

The number of clones a given clone interacts with, its connectivity, is another emergent property of these networks. During early ontogeny, before the network reaches its equilibrium size, the connectivity may become very high. Within a few months however, networks attain a degree of connectivity that is hardly dependent on the matching probability of the receptors. The networks appear to select for specificity: the average connectivity always remains lower than expected, and the selection process favors adding novel specific clones over maintaining established 
multireactive ones. We discuss the "dominance" of specificity, and the fact that the connectivity is lower than expected because clones tend to occupy similar regions in shape space.

The rate at which antibodies in solution are removed from the system by forming idiotypic complexes, and the parameter in the dose-response curve determining the onset of suppression, turn out to be the most crucial parameters of the model.

To summarize, we show how an immune network could select a limited actual repertoire from a seemingly infinite source of novelty from the bone marrow.

\section{Introduction}

Jerne (1974) postulated that the immune system functions as a network with a complexity comparable to that of the neural network. The immune network is based upon so-called "idiotypic" interactions between B lymphocyte clones expressing antibody receptors of complementary shapes. Most previous theoretical studies of idiotypic networks have addressed the existence of multiple steady-states in a network and the phenomenon of immunological memory. Here we study properties of the network such as its size and connectivity, and show via a simulation model that the immune network developed during early ontogeny is capable of influencing the adult immune repertoire. This is in agreement with recent experiments.

The number of immunoglobulin molecules that an animal can make is called its potential repertoire (Holmberg et al., 1986a). Calculated on the basis of the size of the immunoglobulin variable region gene segment libraries and the errors introduced during gene segment joining, the potential repertoire of the mouse has been estimated to be larger than $10^{10}$ (Berek \& Milstein, 1988). The number of different immunoglobulins in an animal used as lymphocyte receptors, its available (or expressed) repertoire, however, is estimated to remain well-below $10^{8}$ (Holmberg et al., 1986a; Berek \& Milstein, 1988). The actual repertoire is composed of antibodies that are being secreted by activated B lymphocytes (Holmberg et al., 1986a). The formation of the available and the actual antibody repertoire is thought to involve several selection mechanisms. One of them is the idiotypic network. The establishment of the adult $\mathrm{B}$ cell repertoire was demonstrated to depend on idiotypic interactions during early ontogeny (Cosenza \& Köhler, 1972; Hiernaux et al., 1981; Kearney \& Vakil, 1986; Vakil et al., 1986). Compared with adult life, idiotypic interactions are very common during early ontogeny (Holmberg et al., 1986b, 1989). Connectivity assays in which a collection of antibodies from unimmunized animals are tested for the ability to react with each other, have suggested that antibodies from newborn animals, on average, match $23-28 \%$ of other antibodies in the collection (Holmberg et al., 1984; Kearney et al., 1987), whereas hardly any antibodies from unimmunized adult animals match each other (Holmberg et al., 1986 b). The pronounced network interactions seen in newborn animals are found among the so-called "natural" antibodies. These antibodies are present in the serum before antigen has been given, they are of the IgM type, and they preferentially use specific V-region gene families (Bona, 1988). A subclass of B cells, identified by the Lyl or CD5 marker, might be responsible for generating these networks of natural antibodies. The $\mathrm{Lyl}^{+} \mathrm{B}$ cells appear during early ontogeny, are multispecific, are probably independent of helper T cells, and play a role in autoimmunity (Hardy \& Hayakawa, 1986; Herzenberg 
et al., 1986). However, in animals exposed to antigen, antibodies of the IgG isotype are made. Hsu et al. (1989) recently found that antigen specific monoclonal IgG antibodies obtained from adult animals immunized with the protein antigen hen egg white lysozyme show a high connectivity. Thus, high network connectivity can be obtained in a variety of circumstances. Here we will focus on networks in unimmunized animals, which appear to be most pronounced in neonatal animals and amongst IgM antibodies (Holmberg et al., 1986b, 1984).

The most detailed data on idiotypic repertoire selection have been obtained with a four-layered (i.e. Ab1-Ab4) network of several perinatal monoclonal IgM antibodies that are known to play a role in the immune response to the antigens phosphorylcholine and dextran (Kearney \& Vakil, 1986; Vakil et al., 1986). The data show that in vivo manupulation of the developing mouse immune system with members of this network influences the adult $\mathrm{B}$ cell response to these antigens. For example, administration of $\mathrm{Ab} 2, \mathrm{Ab} 3$, or $\mathrm{Ab} 4$ antibodies during the first weeks of life enhances or suppresses the adult immune response to phosphorylcholine and dextran depending on the time of administration. The administration of $\mathrm{Ab} 2$ or $\mathrm{Ab} 3$ antibodies can restore the suppressive effect of an earlier dose of an Ab4 antibody. These data show that idiotypic interactions during early ontogeny can influence the adult immune response.

Lundkvist et al. (1989) detected the presence of idiotypic anti-idiotypic interactions among antibodies in unimmunized mice. They found that the concentrations of these natural IgM antibodies fluctuate in a two- to three-fold range. Although they have too little data to be conclusive, Lundkvist et al. (1989) suggest that the concentration fluctuations they measured are compatible with the existence of chaotic dynamical behavior. Treatment of the mice with (probably) low doses of either idiotypic or anti-idiotypic antibody resulted in the inhibition of the fluctuations for months. These results were taken to indicate that there exist functional connections in an idiotypic network of natural $\operatorname{IgM}$ antibodies that are involved in the regulation of antibody concentrations. Additionally, Varela \& Stewart (1990) and Stewart \& Varela (1990), interpreting these data along the lines of their formal model, argue that this oscillatory behavior enables the network to sustain diversity.

According to the view of Vaz \& Varela (1978), Coutinho et al. (1984), and Varela et al. (1988) the immune system is not antigen driven. Rather the system is organized as a network reacting autonomously with itself. The autonomous activity described in the Lundkvist experiment seems to confirm this view. Other experiments, such as the observation that $10-20 \%$ of the lymphocytes from "antigen-free" mice are activated (Hooykaas et al., 1984; Pereira et al., 1986), also suggest autonomous activity that is due to interactions either within the network, or with the environment of self antigens (Holmberg et al., 1984, 1986 b, 1989; Coutinho et al., 1984, 1987; Coutinho, 1989; Huetz et al., 1988). The interactions with self antigens are called "self-assertion", and are postulated to prevent auto-immunity. These considerations are evolving into a new view of the immune system in which both a clonal and a network organization are assumed to coexist. In two recent reviews, Coutinho (1989) and Holmberg et al. (1989) argue that about $10-20 \%$ of clones form an idiotypic network. In their view, the other $80-90 \%$ of clones are disconnected from the network 
and form a clonal compartment of immunocompetent but resting lymphocytes that is responsible for immune responses to foreign antigens. The network is thought to select which $\mathrm{T}$ and $\mathrm{B}$ cell clones are expressed in an animal. This is what we call repertoire selection. We here refer to the two compartments as the "clonal system" and the "immune network".

Here we propose and analyze a model for the development of an immune network. The model will demonstrate how repertoire selection can be carried out by an idiotypic network, and will show how certain features of the network, such as its size and connectivity develop. As our model networks develop, the connectivity first increases, so that each clone reacts with many others, but then decreases so that in adults each clone reacts with rather few species. This developmental pattern follows that observed by Holmberg et al. (1986b).

\section{The Model}

Our model is composed of a varying number of B cell subpopulations of different specificities that form a network. A source, meant to model the bone marrow, supplies novel B cell subpopulations that can be incorporated into the network. Each subpopulation, or clone, is characterized by its specific antibody receptor, which is specified in the model by a bit-pattern that reflects the "shape" of the antibody (cf. Farmer et al., 1986). Two clones can interact via solution phase antibodies whenever their receptor shapes (i.e. bit-patterns) are complementary (Fig. 1). For simplicity, we quantify the degree of match between molecules in terms of


FIG. 1. A diagrammatic representation of some of the interactions incorporated in the model. Each antibody is represented by a bit-pattern, here shown as black and white squares. The rule that 0 is complementary to 1 is illustrated by black being complementary to white. 
only two affinity classes, which can be thought of as high and low. The affinity, $\mathbf{J}_{i j}$, of antibody $j$ for the receptors on cells of clone $i$, is important in determining the degree of stimulation of the B cells of clone $i$. Cells that become activated, proliferate and differentiate into antibody secreting cells. This maturation process takes a few days. Free antibodies may also react with complementary antibodies to form complexes. These complexes, which are analogous to antigen-antibody complexes, will be removed from the system by phagocytic cells such as macrophages. The rate of complex removal will turn out to be one of the most crucial parameters of the model. Each clone is stimulated (and possibly over-stimulated) by the binding of all possible complementary anti-idiotypic antibodies in the network. Their combined effect, denoted $\bar{A}_{i}$, is referred to as the field of clone $i$. This term originates from physics and has been used in neural network theory. Previous authors in idiotypic network theory have called it the connectivity (Hoffmann et al., 1988) or the sensitivity (Varela et al., 1988). We prefer the term field and use it exclusively here. The field of a clone represents the combined effect of all the anti-idiotypic antibodies in the environment that the clone interacts with, weighted by the affinities of the various interactions; i.e.

$$
\overline{A_{i}}=\sum_{j=1}^{n} \mathbf{J}_{i j} A_{j},
$$

where $\mathbf{J}_{i j}$ defines the affinity with which antibody $j$ interacts with clone $i$.

As we shall see below, our model also includes direct interactions between antibody $i$ and antibody $j$. For simplicity, we assume that the affinity of antibody $j$ for antibody $i$ is the same as its affinity for a receptor $i$. Thus, in this simplification we are not distinguishing between cell surface antibody and solution antibody with regard to their binding properties. Because of this, it is natural to assume that the affinity matrix $\mathbf{J}$ is symmetric, i.e. $\mathbf{J}_{i j}=\mathbf{J}_{j i}$. If we were to distinguish between these two classes of reactions, as in Segel \& Perelson (1989), one might argue that because of the environment of a receptor on a cell surface (e.g. charge and steric hindrance effects due to other cell surface moieties) it would be possible to have a nonsymmetric affinity matrix characterizing the reactions between solution phase antibodies and cell surface receptors. Non-symmetric matrices do occur in ELISA measurements of antibody connectivity (Kearney et al., 1987; Hsu et al., 1989).

To model the dynamics of the various B cell clones we consider that cells emerge from the bone marrow at rate $m$, proliferate at rate $p$ upon idiotypic stimulation, and decay at rate $d_{B}$. Thus

$$
\frac{\mathrm{d} B_{i}}{\mathrm{~d} t}=m+B_{i}\left[p f\left(\overline{A_{i}}\right)-d_{\mathrm{B}}\right],
$$

where $f\left(\bar{A}_{i}\right)$ is the fraction of activated $B$ cells of type $i$. The activation function $f$, assumed to be the same for all clones $i$, depends only on the field seen by the clone.

The most crucial assumption of this model is the shape of the activation function. Activation is based upon a biphasic dose-response function

$$
f\left(\bar{A}_{i}\right)=\frac{\bar{A}_{i}^{\eta}}{\theta_{1}^{\eta}+\bar{A}_{i}^{\eta}} \frac{\theta_{2}^{\eta}}{\theta_{2}^{\eta}+\bar{A}_{i}^{\eta}},
$$


which depends on three parameters, $\eta, \theta_{1}$, and $\theta_{2}$. This function has been proposed previously (De Boer, 1988; De Boer \& Hogeweg, 1989a). We choose $\theta_{2} \gg \theta_{1}$, so that the function is the product of two well-separated sigmoid functions, one increasing from 0 to 1 , reaching its half-maximal value at $\theta_{1}$, the other decreasing from 1 to 0 , reaching its half-maximal value at $\theta_{2}$. The steepness of the sigmoidal functions is determined by the "Hill" coefficient $\eta \geqslant 1$. For large values of $\eta$ the activation function switches over a small range of $\overline{A_{i}}$ values centered at $\theta_{1}$ from near 0 to near 1 , and at values centered at $\theta_{2}$ from near 1 to near 0 . The parameters, $\theta_{1}$ and $\theta_{2}$ are called thresholds and in essence define the field values required for half-maximum proliferation and suppression, respectively. Note that $0 \leq f(\cdot) \leq f_{\max }$ where $f_{\max }=\theta_{2}^{\eta} /\left(\theta_{1}^{\eta / 2}+\theta_{2}^{\eta / 2}\right)^{2}$, and that, for $\theta_{2} \gg \theta_{1}, f_{\max } \approx 1$. The maximum is reached at $\overline{A_{i}}=\sqrt{\theta_{1} \theta_{2}}$. Additionally, note that if $f\left(\bar{A}_{i}\right)$ is plotted against $\log \left(\bar{A}_{i}\right)$ the graph is symmetric around its maximum. Further, for $\theta_{2} \gg \theta_{1}$, at each of the thresholds $f(\theta) \approx 0 \cdot 5$. Due to the biphasic nature of the activation function, high concentrations are suppressive. Here we use the term suppression to mean that increasing the stimulus decreases the response. In other words, suppression is a form of over-stimulation in our model. An antibody is suppressive whenever its population is larger than $\sqrt{\theta_{1} \theta_{2}}$. Note that, because $\theta_{2} \gg \theta_{1}$, the "threshold for suppression" $\theta_{2}$ is larger than $\sqrt{\theta_{1} \theta_{2}}$.

Plotted as a function of $\log \bar{A}_{1}$, the graph of $f\left(\bar{A}_{i}\right)$ is a bell-shaped curve, the steepness of which depends on $\eta$. An important argument for the use of a log bell-shaped function is that receptor crosslinking is involved in B cell activation. The fraction of cell surface receptors cross-linked by a ligand when plotted against the logarithm of the ligand concentration is called a crosslinking curve. For ligands that are bivalent the crosslinking curve is bell-shaped and symmetric around its maximum (Perelson \& DeLisi, 1980; Perelson, 1984). Indeed, the behavior of this model resembles the behavior of more complicated models that incorporate the chemistry involved in receptor binding and crosslinking (Perelson, 1989a; De Boer et al., 1990). For reasons of simplicity, we shall study eqn (1) for $\eta=1$. For $\eta=1$, this function has been studied extensively (De Boer \& Hogeweg, 1989a, $b$; De Boer et al., 1990; Weisbuch et al., 1990), and accounts for interesting network behavior. Varela et al. (1988) have also proposed biphasic activation functions giving rise to similar network behavior; see also the discussion in Coutinho (1989). They, however, use two biphasic functions: one for proliferation and one for maturation. Our model accounts for maturation by incorporating a simple one compartment model for a time-delay.

B cells that are stimulated produce antibodies at a rate $s(t)$. To model the timedependent aspects of antibody production we introduce a "gearing up" function, $G(t)$, that accounts for the time lag associated with the differentiation of small stimulated B cells to the large lymphocyte and plasma cell states that are characteristic of antibody secreting cells. Thus, we set the rate of antibody secretion $s(t)=s G(t)$, where $s$ is a constant equal to the maximal secretion rate and the function $G(t)$ is chosen such that $0 \leq G(t) \leq 1$. We introduce a separate gearing up function for each clone so that $G_{i}(t)$ can be interpreted as the proportion of mature B cells of type $i$. Following Segel \& Perelson (1989) we construct $G_{i}(t)$ as the solution of the 
first-order differential equation

$$
\frac{\mathrm{d} G_{i}}{\mathrm{~d} t}=k\left[f\left(\bar{A}_{i}\right)-G_{i}\right]
$$

where $f\left(\bar{A}_{i}\right)$ is the activation function and $k$ is a constant that determines the characteristic time for gearing up. At $t=0$ we set $G_{i}(0)=0$ so so that there is no initial secretion. When a clone becomes activated, $f\left(\bar{A}_{i}\right)$ increases from 0 to a positive value and $G(t)$ increases. If $\bar{a}_{i}$ where constant, $G(t)$ would increase until $G_{i}=f\left(\bar{A}_{i}\right)$. However, as $A_{i}$ changes in time $G(t)$ follows with a lag. This is illustrated in Fig. 2(b). Other authors, e.g. Dibrov et al. (1987) and Fowler (1981), explicitly introduce a time delay $\tau$ such that the antibody production at time $t$ depends on the $\mathrm{B}$ cell population, and its degree of stimulation, at a time $t-\tau$ in the past. However, since antibody production at time $t$ depends on the B cells present at time $t$, our model seems more appropriate.

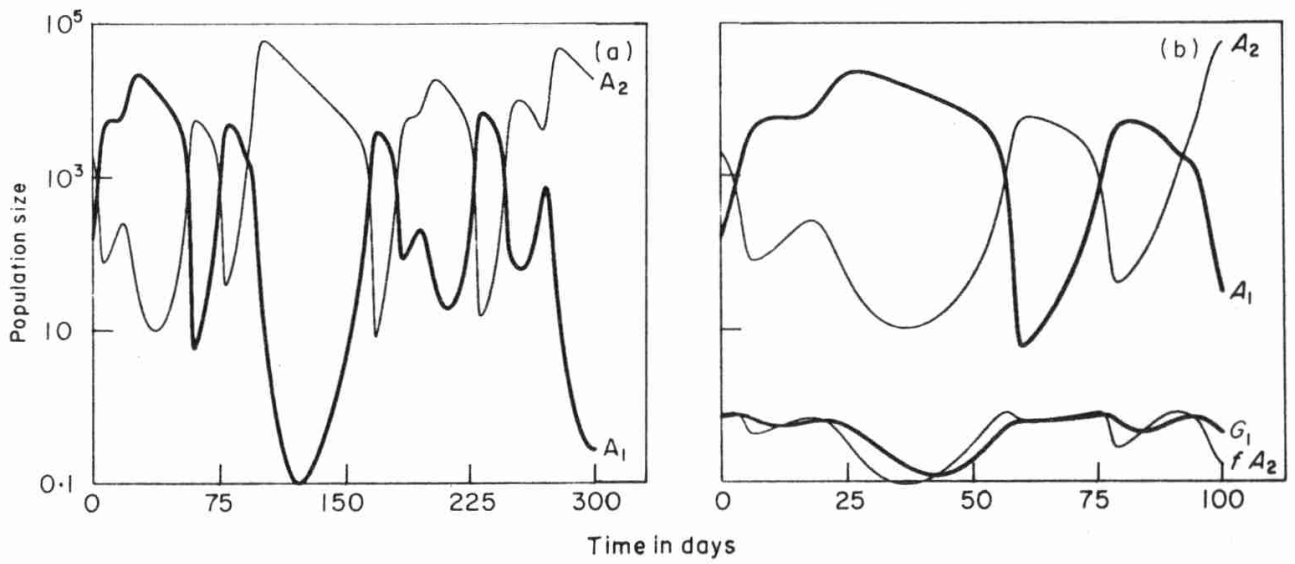

FIG. 2. Possible dynamic behavior of a network of two clones following an immune response to a stimulatory dose of $\boldsymbol{A}_{2}$. Parameters: $d_{C}=10^{-3}$ and $m=1$. (a) A plot of $\boldsymbol{A}_{i}$ vs. $t$ for the chaotic attractor of Fig. 3(f) displaying periods of "quiescence". (b) The "gearing up" function: the relation between $A_{1}$, $A_{2}, f\left(\bar{A}_{1}\right)=f\left(A_{2}\right)$, and $G_{1}$ during an immune response to a stimulatory dose of $A_{2}$.

After antibodies are secreted they are free in solution. Free antibodies can form complexes with complementary antibodies and be eliminated at rate $d_{C}$. Additionally, antibodies decay at rate $d_{A}$. Thus,

$$
\frac{\mathrm{d} A_{i}}{\mathrm{~d} t}=s B_{i} G_{i}-A_{i}\left(d_{C} \bar{A}_{i}+d_{A}\right) \text {. }
$$

The formation of an antibody-antibody complex is a reversible reaction, which typically reaches equilibrium rather rapidly, i.e. on a time scale of seconds to minutes. Thus, on the time scale of days, appropriate to cellular changes these reactions should be thought of as being at equilibrium. If this is the case, then the concentration of $A_{i}-A_{j}$ complex is $\mathbf{J}_{i j} A_{i} A_{j}$. Thus, the coefficient $d_{C}$ multiplies the equilibrium concentration of all possible complexes, i.e. $A_{i} \sum_{j} \mathbf{J}_{i j} A_{j}$, see Appendix. 
To summarize, our model can be formally described by the following system of equations:

$$
\begin{gathered}
f\left(\bar{A}_{i}\right)=\frac{\overline{A_{i}}}{\theta_{1}+\bar{A}_{i}} \frac{\theta_{2}}{\theta_{2}+\bar{A}_{i}} \\
\bar{A}_{i}=\sum_{j=1}^{n} \mathbf{J}_{i j} A_{j} \\
\frac{\mathrm{d} B_{i}}{\mathrm{~d} t}=m+B_{i}\left[p f\left(\overline{A_{i}}\right)-d_{B}\right] \\
\frac{\mathrm{d} A_{i}}{\mathrm{~d} t}=s B_{i} G_{i}-A_{i}\left(d_{C} \bar{A}_{i}+d_{A}\right) \\
\frac{\mathrm{d} G_{i}}{\mathrm{~d} t}=k\left[f\left(\bar{A}_{i}\right)-G_{i}\right] .
\end{gathered}
$$

It consists of $3 n$ differential equations, where $n$ is the number of clones in the system. For each idiotype $i$ there is one equation for the $\mathrm{B}$ cells $\left(B_{i}\right)$, one for the antibodies $\left(A_{i}\right)$, and one for the maturation of $\mathrm{B}$ cells to antibody secretion, i.e. the gearing up process $\left(G_{i}\right)$. Additionally, for each idiotype we compute the field due to complementary antibodies $\left(\overline{A_{i}}\right)$. These fields are determined by summing the concentration of all antibodies weighted by their affinity with the antibody of clone $i$.

This model is a combination of two previous ones. The B cell equation, the antibody equation, and the activation function were previously defined by (De Boer, 1988; De Boer \& Hogeweg, 1989a, b; De Boer et al., 1990; Weisbuch et al., 1990). The gearing up function is the one defined previously by Segel \& Perelson (1989) and Perelson (1989a).

\section{PARAMETERS}

Most of the parameter values were presented before (De Boer \& Hogeweg, $1989 a, b)$. For each clone, the rate $m$ at which cells appear from the bone marrow should be small. We either choose $m=1$ or $m=0$. The values of the matrix $\mathbf{J}$ are either $0,0 \cdot 1$ or $1 \cdot 0$, denoting no interaction, a low affinity interaction, and a maximum affinity interaction respectively. The Hill coefficient $\eta$ describing the steepness of the bell-shaped activation function has been set to one and hence does not appear in eqn (1). The population of antibody molecules is normalized so that each B cell produces maximally 1 unit of antibody/day: $s=1$ day $^{-1}$. If we assume a maximal secretion rate of 10000 antibodies per second, then one unit corresponds to $1.4 \times$ $10^{-15}$ moles of antibody. B cells have a lifetime of 1-2 days: $d_{B}=0.5 \mathrm{day}^{-1}$. Antibodies are known to persist much longer; we chose $d_{A}=0.05$ day $^{-1}$ (i.e. about 20 days). B cell division (proliferation) takes about $16 \mathrm{hr}$; in the model each $\mathrm{B}$ cell proliferates maximally at a net rate $p-d_{B}$. In order to account for a $16 \mathrm{hr}$ cell cycle 
$p=1$ day $^{-1}$. The onset of proliferation (i.e. $\theta_{1}$ ) is kept low (i.e. $\theta_{1}=100$ ) in order to facilitate "natural" idiotypic interactions (cf. De Boer \& Hogeweg, 1989b). The onset of "suppression" (i.e. $\theta_{2}$ ) determines the maximum B cell population; we chose $\theta_{2}=10^{4}$. In order to account for considerable clonal expansion these thresholds should be several orders of magnitude apart (i.e. $\theta_{2} \gg \theta_{1}$ ). At either threshold $f(\theta)=0.5$. Because $p=1$ day $^{-1}$ and $d_{B}=0.5$ day $^{-1}$, at threshold $p f\left(\bar{A}_{i}\right)-d_{B}=0$. The source $m$ is small compared with the production of cells at proliferation, thus to a good approximation, the B cell populations attain an equilibrium whenever $\bar{A}_{i} \simeq \theta_{1}$ or $\theta_{2}$ (De Boer \& Hogeweg, 1989a, b; De Boer et al., 1990; Weisbuch et al., 1990). The gearing up parameter $k=0 \cdot 2$ day $^{-1}$ sets a time scale for antibody production of about 5 days. The rates at which antibodies are removed by complex formation $\left(d_{C}\right)$ is largely unknown. The $d_{C}$ parameter is varied throughout the analysis; the typical range of $d_{C}$ values is discussed in the Appendix. For simplicity parameter values will be quoted with their units omitted. The time unit is always day ${ }^{-1}$.

\section{Simulation}

We study the model defined by eqns (1-5) in two distinct ways. First, we examine a simple example containing only two clones that are complementary to each other. Using numerical solution of the ordinary differential equations, employing the program GRIND (De Boer, 1983), coupled with analysis of the steady-states and numerical bifurcation techniques, using AUTO (Doedel, 1981), we build up a detailed picture of the dynamical behaviors inherent in our description of $\mathrm{B}$ cell proliferation, differentiation and antibody interactions. This work provides a baseline for our subsequent study of a large dynamically evolving network. The study of systems containing only two clones that interact through solution phase antibody has been studied previously by (Perelson, 1989a; De Boer et al., 1990; Stewart \& Varela, 1990). What distinguishes the work presented here is the particular form of the differential equations under study. In Perelson (1989a) a system of 11 differential equations was used and the interactions between cells and molecules was described in great chemical detail. In our previous work, (De Boer et al., 1990) we ignored gearing up, which reduced the system to four differential equations. In the next section, after our study of the two clone system is completed we present our model for the in vivo development of idiotypic networks. Rather than prespecifying the components of the network, we characterize each clone by a bit-string and use a string matching algorithm to determine when a clone has a receptor complementary to a solution phase antibody. No constant source is included (i.e. $m=0$ ), but rather new clones are generated and injected into the system at rate $M$.

\section{Dynamics of Two Interacting Clones}

Consider a network of two populations, $i=1,2$, that interact with each other with affinity $\mathbf{J}_{12}=1$. We exclude self interactions and thus $\mathbf{J}_{11}=\mathbf{J}_{22}=0$. New cells are supplied from the bone marrow to each clone at rate $m=1$ day $^{-1}$. The balance 
between the supply of new cells and the turnover of cells generates a stable virgin state that is devoid of idiotypic stimulation (i.e. $B_{i}=m / d_{B}$ ). The transition from this virgin state to immune states has been the topic of previous studies (De Boer, 1988; De Boer \& Hogeweg, 1989a, b; Weisbuch et al., 1990). In Fig. 3 we depict the behavior of the model projected in the $A_{1}-A_{2}$ phase-plane. The behavior depicted is attained for a network in the virgin state stimulated by the introduction of either of the two antibodies in a maximum stimulatory concentration (i.e. $A_{i}=\sqrt{\theta_{1} \theta_{2}}=10^{3}$ ). Varying the rate of complex elimination, $d_{C}$, we found several types of oscillatory

(a)
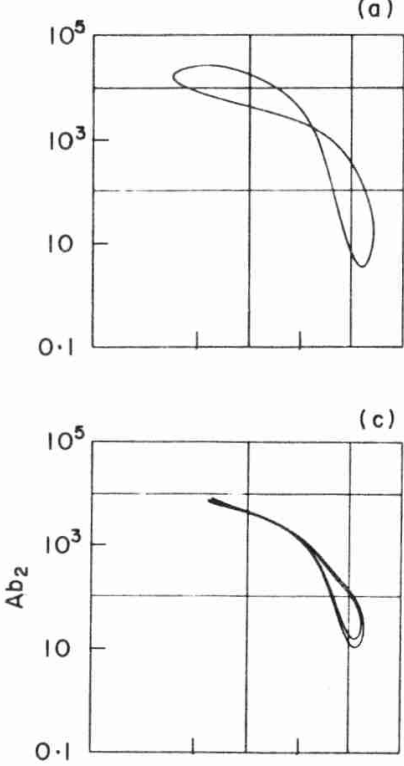

(e)

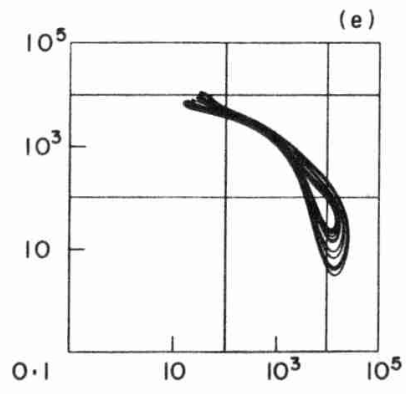

(b)

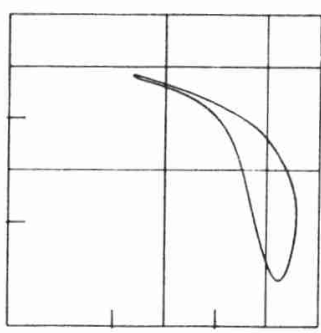

(d)

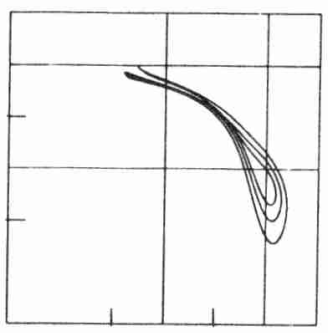

(f)

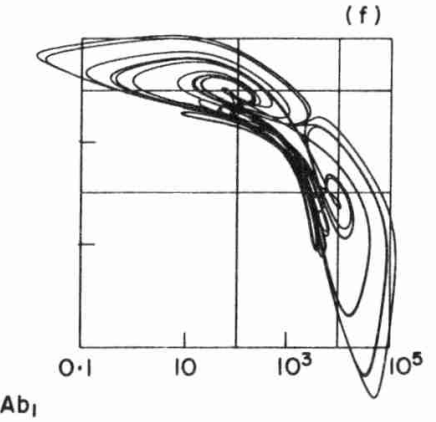

FIG. 3. Possible behaviors of a network of two clones examined by varying the rate of complex formation, $d_{C}$. In each panel we plot $A_{1}$ vs. $A_{2}$ on a logarithmic scale. Parameters: $m=1, J_{12}=J_{21}=1$, and $\mathbf{J}_{11}=\mathbf{J}_{22}=0, \theta_{1}=10^{2}$ and $\theta_{2}=10^{4}$. The straight lines are $A_{i} \simeq \theta_{1}, \theta_{2}, i=1,2$. (a) Symmetric periodic behavior for $d_{C}=10^{-4}$. (b) Asymmetric periodic behavior for $d_{C}=4 \times 10^{-4}$. (c, d) Period doubled asymmetric cycles for $d_{C}=4 \cdot 7 \times 10^{-4}$ and $d_{C}=5 \times 10^{-4}$. (e) Noisy periodicity for $d_{C}=5 \cdot 2 \times 10^{-4}$. (f) Symmetric chaotic attractor $d_{C}=10^{-3}$. 
behavior [Fig. 2(a) and Fig. 3(a)-(f)]. The trajectories fluctuate around the equilibria that are present when both antibody populations equal $\theta_{1}$ and/or $\theta_{2}$ (see the straight lines in the Fig. 3, which in fact are the $B_{1}$ and the $B_{2}$ nullclines for $m=0$ ).

Figure 3 (a) shows an example of a symmetric cycle (e.g. $d_{C}=10^{-4}$ ) which gives rise to two asymmetric cycles by a pitchfork bifurcation. An example of one of them is depicted in Fig. 3(b) (for $d_{C}=4 \times 10^{-4}$ ). The asymmetric cycles go through period doublings [e.g. Fig. 3(c), $d_{C}=4.7 \times 10^{-4}$ and Fig. 3(d), $d_{C}=5 \times 10^{-4}$ ], display "noisy periodicity" [e.g. Fig. $\left.3(\mathrm{e}), d_{C}=5 \cdot 2 \times 10^{-4}\right]$, and give rise to a chaotic attractor [e.g. Figures 2(a) and 3(f), $d_{C}=10^{-3}$ ] which appears similar to the Lorenz attractor. The sustained fluctuating behavior disappears at even larger rates of complex formation (e.g. $d_{C}=10^{-2}$ ): following some oscillations the system returns to the virgin state. Immunologically, we conclude that the behavior of this model is typically oscillatory and/or chaotic. The concentrations of complementary antibody appear to be correlated inversely; see Fig. 3. Changing $d_{C}$ we find several windows in which periodic and chaotic solutions alternate. As suggested by these examples, we also find the classical cascades of symmetry breaking, period doublings, and eventually chaos. In this respect, and visually, this chaotic attractor is similar to the Lorenz attractor (Lorenz, 1963; Sparrow, 1982). These, and other, properties were described in much more detail for a simplified version of this model which omits the time-delay (De Boer et al., 1990).

Two stable equilibria, corresponding to "immune" ( $B_{1}$ high, $B_{2}$ low) and "suppressed" ( $B_{1}$ low, $B_{2}$ high) states, arise in the two-clone networks for what we believe to be unrealistic parameter values. An example would be $d_{C}=0 \cdot 01, k=10$, i.e. rapid complex elimination and rapid maturation. These equilibria can only be stable if maturation is sufficiently fast (i.e. $k>5$, not shown). The presence of stable equilibria may account for idiotypic memory (De Boer \& Hogeweg, 1989 b; Weisbuch et al., 1990). For the parameter choices we made in this paper stable immune states are absent and the behavior of the model is typically oscillatory.

\section{QUIESCENT PERIODS}

As a part of the normal chaotic behavior one observes time intervals during which one of the antibody concentrations is large, and is decaying exponentially, while the other antibody has almost disappeared. For example, in Fig. 2(a) we see this behavior with $A_{1}$ large and $A_{2}$ small for $t=80-150$ days. When $A_{1}$ is large, population $A_{2}$ remains small because $B_{2}$ cells are suppressed by the high field caused by $A_{1}$ and thus they fail to produce antibody. Additionally, $A_{2}$ is removed by complex formation with the large amount of $A_{1}$. In the absence of $A_{2}, B_{1}$ cells remain unstimulated and thus $A_{1}$ is not being produced and chemical interactions between $A_{1}$ and $A_{2}$ are negligible. Thus, the dominant effect is that the large antibody population, $A_{1}$, decays exponentially by normal turnover at a rate $d_{A}$. Ultimately, the concentration of $A_{1}$ falls into the stimulatory regime, leading to the renewed production of $\boldsymbol{A}_{2}$ and fluctuations resume. This situation can arise whenever one antibody concentration becomes large, resulting in the removal of its complementary antibodies by complex formation. We call this type of dynamical behavior quiescent 
because the fluctuations cease. Because one of the antibodies severely suppresses the other, one could also speak of dominance of one of the two. We think that quiescent behavior develops whenever a trajectory approaches the inset of the symmetric saddle equilibrium in which $A_{1}=A_{2} \simeq \theta_{2}$ [see Fig. 3 and De Boer et al. (1990)]. The one-dimensional outset of this equilibrium pushes such trajectories into the regions where either $A_{1} \gg A_{2}$ or $A_{2} \gg A_{1}$ (De Boer et al., 1990). This again resembles the Lorenz attractor where the largest amplitudes are found following the approach of the symmetric saddle state along its inset. In our model the length of the quiescent periods depends on the rate of antibody decay $\left(d_{A}\right)$, and the size of the large antibody population.

Our model predicts that quiescent periods can be induced in vivo by injecting a sufficient amount of one of two complementary antibodies. An experiment of this type has been performed in mice by Lindkvist et al. (1989) (see Introduction) and results in a cessation of apparent chaotic behavior. Our interpretation of this experiment is described in greater detail in De Boer et al. (1990).

\section{PERIODS}

The limit cycle displayed in Fig. 3(a) has a period of 115 days. If one keeps the rate of complex formation at $d_{C}=10^{-4}$ and varies the antibody decay rate $d_{A}$, one finds that the period of the cycle depends linearly on the antibody lifetime, $1 / d_{A}$. Within the biologically reasonable range $0.01 \leq d_{A} \leq 0.08$ the period length ranges from 83-520 days, which can be fitted to $15+5 / d_{A}$ days. Above $d_{A}=0.08$ we found a symmetry breaking (pitchfork) bifurcation, period doublings and chaos (not shown).

By means of a Fast Fourier Transform (Press et al., 1988), we computed power spectra of the fluctuations found in the chaotic regimes corresponding to several rates of complex formation $\left(d_{C}\right)$. The final power spectrum was based upon the average of five individual spectra, each computed from a time series of 1024 days. These spectra were typically $1 / f^{\beta}$, with $\beta \simeq 3$. In addition to the low frequencies that are typical of chaos, we found several minor peaks that depended on $d_{C}$. For $d_{C} \leq 10^{-3}$, these peaks typically correspond to periods between 30 and 150 days. This is in agreement with empirical data of Rodkey \& Adler (1983) which shows an 80-day period in rabbits. Networks with larger rates of complex formation failed to have sustained oscillatory behavior. This can be circumvented by increasing the bone marrow production $m$, but one finds different periods for different values of $m$ (not shown). A number of these (e.g. $m=50$ ), have the 2-4-week period that was suggested by the in vitro experiments by Kelsoe \& Cerny (1979) and the in vivo experiments by Lundkvist et al. (1989). The fact that the period becomes shorter if the rate of antibody removal by complex formation increases again suggests that it is the antibody lifetime (as compared to the B cell lifetime) which determines the periods.

We conclude that the time-scale of the chaotic/oscillatory behavior depends on the lifetime of the antibodies, which in turn is determined by the rate of normal turnover $d_{A}$ and the rate of complex formation $d_{C}$. Since $d_{C}$ varies with the affinity 
of the antibodies (see Appendix), our model is able to account for differences in time scales of oscillations in different experimental systems, e.g. Kelsoe \& Cerny (1979), Lundkvist et al. (1989), and Rodkey \& Adler (1983), if different antibody affinities are involved.

\section{Development of Idiotypic Networks}

Having described the basic dynamics of idiotype anti-idiotype clones, we now examine a more realistic model in which clones are generated at random; if they are successful in the sense that the cells in the clone are stimulated to grow the clone remains in the system, otherwise it dies out. This model will exhibit a number of interesting features. First, the network will develop to some characteristic size, and then despite the constant generation of new clones will fail to grow any larger. We will characterize the equilibrium size of the emergent network in terms of the rate of generation of novel clones, a parameter $P$ (match), and the various parameters such as $d_{C}$ introduced in the two clone model. By $P$ (match) we mean the probability that two randomly generated antibody bit-strings match each other. $P$ (match) is to be distinguished from the connectivity, which is a network property and defined to be the average number of connections per clone in the network. The two may be different because of selection processes. We will show that networks evolve so as to maintain a characteristic degree of connectivity that is relatively independent of the antibody matching probability. We begin by describing our model.

\section{GENERATION OF NOVEL CLONES}

From the genetics of antibody $\mathrm{V}$ region gene recombination it seems that the potential repertoire should be much greater than the repertoire present in the $B$ cells of an organism at any one time. Typical estimates of the potential repertoire, ignoring somatic mutation, are of order $10^{10}-10^{11}$ (Darnell et al., 1986; Berek \& Milstein, 1988). A mouse with a total of $10^{8} \mathrm{~B}$ lymphocytes should have an available repertoire that is well-below $10^{8}$ (Holmberg et al., 1986 $a$; Berek \& Milstein, 1988). Because of this large discrepancy in repertoire sizes, we shall assume that each time a clone is generated in the bone marrow it contains a unique antibody variable region that is produced for the first time. To account for the total production of cells by the bone marrow, we assume $M$ novel clones are produced daily, with each clone containing about ten cells. We introduce more than 1 cell/clone because of some clonal expansion at the pre-B cell stage (Opstelten \& Osmond, 1983). Once a clone has been generated, we assume that there is no further generation from the bone marrow of cells of that clone, i.e. we assume $m=0$. Using the string matching algorithm described below, each newly generated clone is compared with all other clones already existing in the network. A new clone is incorporated in the network only if (1) it recognizes at least one other clone (or antigen) already present in the network, and if (2) these interactions are sufficiently stimulatory so that the clone is expected to increase following its introduction (i.e. $d B_{i} / \mathrm{d} t>0$ ). Rule (1) restricts 
us to considering only those clones that connect to the network. Coutinho (1989) and Holmberg et al. (1989) have suggested that only $10-20 \%$ of clones participate in the network; the remainder forming a set of independent clones acting according to a classical clonal organization. Rule (2) is just a short-cut to avoid incorporating clones into the network that decrease and have to be removed within a few time steps. The number of clones not connected to the network due to rule (1) or (2) is kept track of, and will play an important role in our discussion of the Coutinho/Holmberg hypothesis of a network and clonal components to the immune system.

\section{CLONE REMOVAL}

At time intervals of one day all clones in the model are checked for the size of the B cell and antibody population. If $B_{i}<1$ and $A_{i}<\theta_{1} / 10$, i.e. if the clone consists of less than one cell, and its antibody population is too small to have any effect, the clone is removed from the network.

\section{MATCHING RULES}

The shape of each antibody molecule is represented as a bit-string of length $L$. Antibody molecules are assumed to recognize each other whenever their bit-strings can be matched complementarily. Such a matching rule is symmetric: if clone " $i$ " matches clone " $j "$, " $j$ " also matches " $i$ " (cf. Hoffmann, 1975). Similar rules have been applied before (Farmer et al., 1986; De Boer, 1988; De Boer \& Hogeweg, $1989 \mathrm{~b})$; and have been discussed in detail by Perelson $(1988,1989 b)$. Stewart \& Varela (1989) have used sine-functions to represent antibody shapes. Weinand (1990) has developed a three-dimensional shape space model in which antibody shapes are represented using unit cubes placed on a grid. The specific rule that we apply here is that we align the bit-strings and require a complementary match over a stretch of at least $T$ adjacent positions. Because the strings are aligned they cannot match themselves (i.e. we exclude autobodies; see Discussion). If the strings match over exactly $T$ adjacent positions we assign a low affinity, i.e. $\mathbf{J}_{i j}=0 \cdot 1$. Whenever the strings match over more than $T$ adjacent positions we assign a high affinity, i.e. $\boldsymbol{J}_{i j}=1 \cdot 0$. Since $L \gg T$ there are several positions on the string to find an adjacent match. This makes it possible to have several independent idiotopes on one idiotype, which generates considerable branching in the tree representing the network topology; see Perelson (1988) and De Boer \& Hogeweg (1989b).

We set $L=32$, and vary $T$ in order to vary the matching probability, $P$ (match), of the receptors. In fact, for our specific match rule, $P$ (match) is the probability of finding a "success run" of length $T$ in a sequence of $L$ Bernoulli trials (e.g. $T$ "heads" in a row in a sequence of $L$ coin tosses). Feller (1978) provides an approximate formula for $P$ (match) that becomes reasonably accurate for $L \geq 2$. Using Feller's result we find for $L=32$ and $T=6,7,8,9,10$, and $11, P$ (match) $=$ $0.205,0 \cdot 103,0.05,0.024,0.012$, and 0.005 respectively. 


\section{INITIAL CONDITIONS}

During the development of a mammal maternal antibodies pass across the placenta, and influence the development of the embyro's immune system. To mimic this effect, we initialized our model with $1 / P($ match) randomly made "maternal antibodies". The concentrations of these antibodies were set to random values in the stimulatory range, i.e. to $\sqrt{\theta_{1} \theta_{2}}$ with $10 \%$ standard deviation; the corresponding $\mathrm{B}$ cell populations and gearing up functions were set to zero. The maternal antibodies were assumed not to interact idiotypically (this was achieved by selecting idiotypes that did not match amongst each other). The idea behind this approach was to generate an initial population that spanned a diversity of initial shapes. The number of clones in this population was made proportional to $1 / P$ (match) because $P$ (match) determines how large a part of the shape space each antibody covers (see below). Because the maternal antibodies are introduced without $\mathrm{B}$ cells, they decay at rate $d_{A}$ and disappear from the system.

\section{SIMULATION}

The topology and dynamics of these large networks were studied by computer simulation via a program coded in the $\mathrm{C}$ programming language. The $\mathrm{C}$ code implements the variable time step Runge-Kutta integrator provided by Press et al. (1988). Each network was simulated for a time corresponding to at least 300 days on a SUN4 Sparcstation 1. Simulations took between 2 and $36 \mathrm{hr}$ of computer time; the longer simulations corresponding to larger values of $M$ and hence larger networks, or to large values of $d_{C}$ or $\theta_{2}$ which turned out to increase the stiffness of the equations.

\section{Results}

In Fig. 4 we plot the dynamics of all the clones in one representative network. We let $L=32$ and $T=8$, for which $P($ match $)=0 \cdot 05$. The bone marrow of the network displayed in Fig. 4 supplies ten novel clones per day (i.e. $M=10$ ). During its lifetime of 300 days, in which period 3000 clones were generated, the network encompassed a total of 933 clones. This corresponds to the 933 separate panels displayed in Fig. 4. Each panel in Fig. 4 shows the antibody population as a function of time for one clone (i.e. $A_{i}$ vs. $t$ ); each panel in Fig. 5 is a projection onto a phase-plane in which the antibody concentration and the corresponding $\mathrm{B}$ cell populations are plotted (i.e. $A_{i}$ vs $B_{i}$ ). Because the $A_{i}$ vs. $B_{i}$ graphs are square, whereas the $A_{i}$ vs. $t$ graphs are elongated, the number of columns in Figs 4 and 5 are not the same, and thus the panels do not line up. Notice that the time plots in Fig. 4 start at different values of $t$, corresponding to the time the clone entered the network. Similarly the plots end at the time the clone exits the network. The panels in Figs 4 and 5 are sorted by the life-average connectivity of the clones: from the left to the right and from the top to the bottom the life-average of the number of connections to other clones increases. The connectivity with which each row starts is indicated on the left in each figure. Thus, in Figs 4 and 5, the clone displayed in the panel at the top-left 

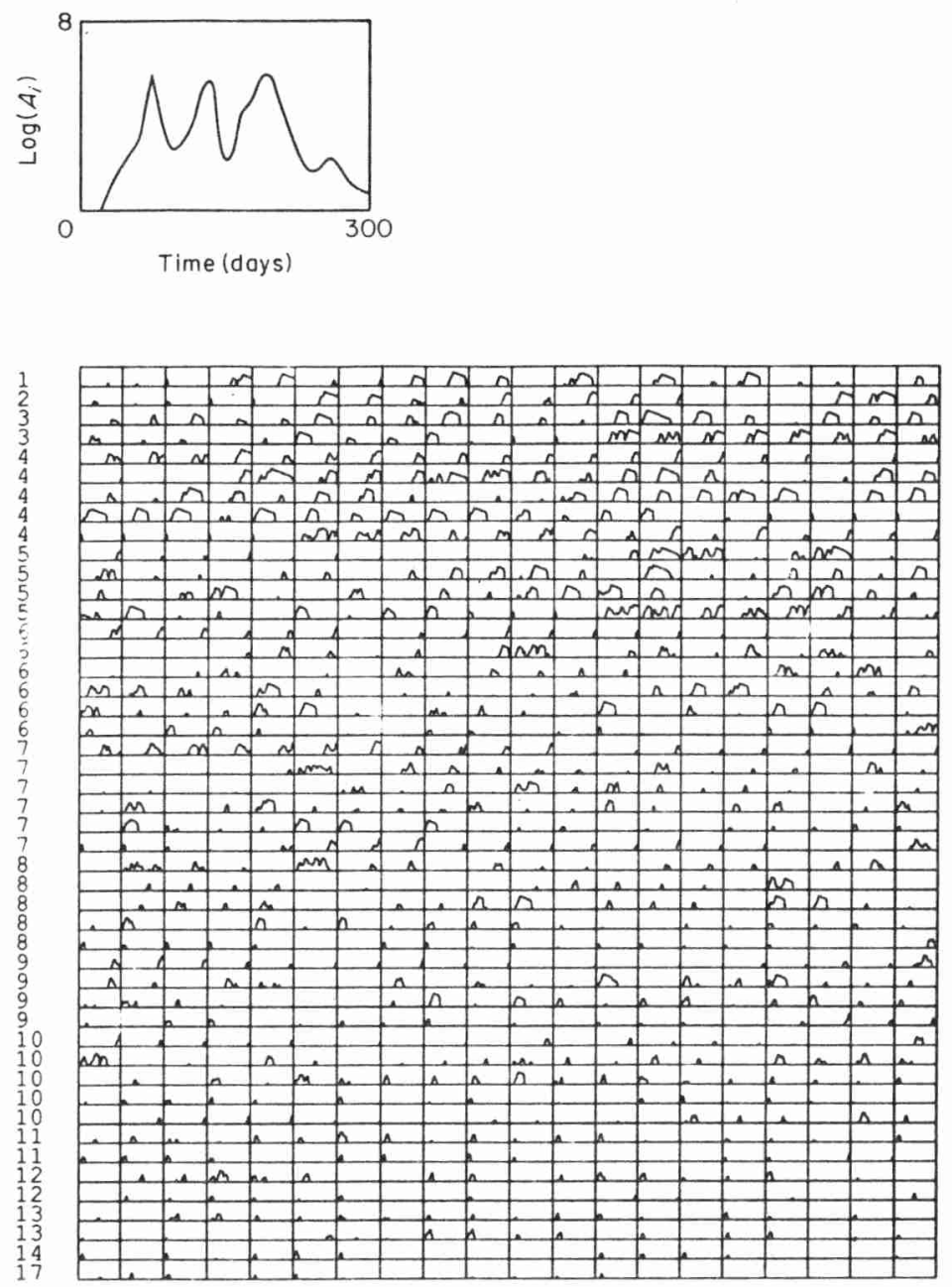

FIG. 4. Dynamic behavior of the 933 clones that were members of the network generated with $d_{C}=10^{-3}$, $\theta_{2}=10^{4}, M=10, T=8, P($ match $)=0 \cdot 05$. Clones are sorted by connectivity (see the text). The time axes runs from zero to 300 days. We plot $A_{i}$ as a function of time. The vertical axis is logarithmic (base 10) and runs from 1 to $10^{8}$. Clones may appear at any point in time, after which they can be seen to expand. Clones disappear when the antibody population is $\theta_{1} / 10=10$, which is just above the horizontal axis.

corner has the lowest connectivity (i.e. one clone); whereas the one in the bottomright corner has the highest connectivity (i.e. 20 clones). By scanning Figs 4 and 5 from the top to the bottom one can examine the effect of a clone's connectivity on its behavior.

In many models of idiotypic networks (e.g. Hoffmann, 1975; Richter, 1975, 1978; Gunther \& Hoffmann, 1982; Hoffmann et al., 1988; Farmer et al., 1986; De Boer, 1988; De Boer \& Hogeweg, 1989a; Segel \& Perelson, 1988; Weisbuch, 1990) it has 

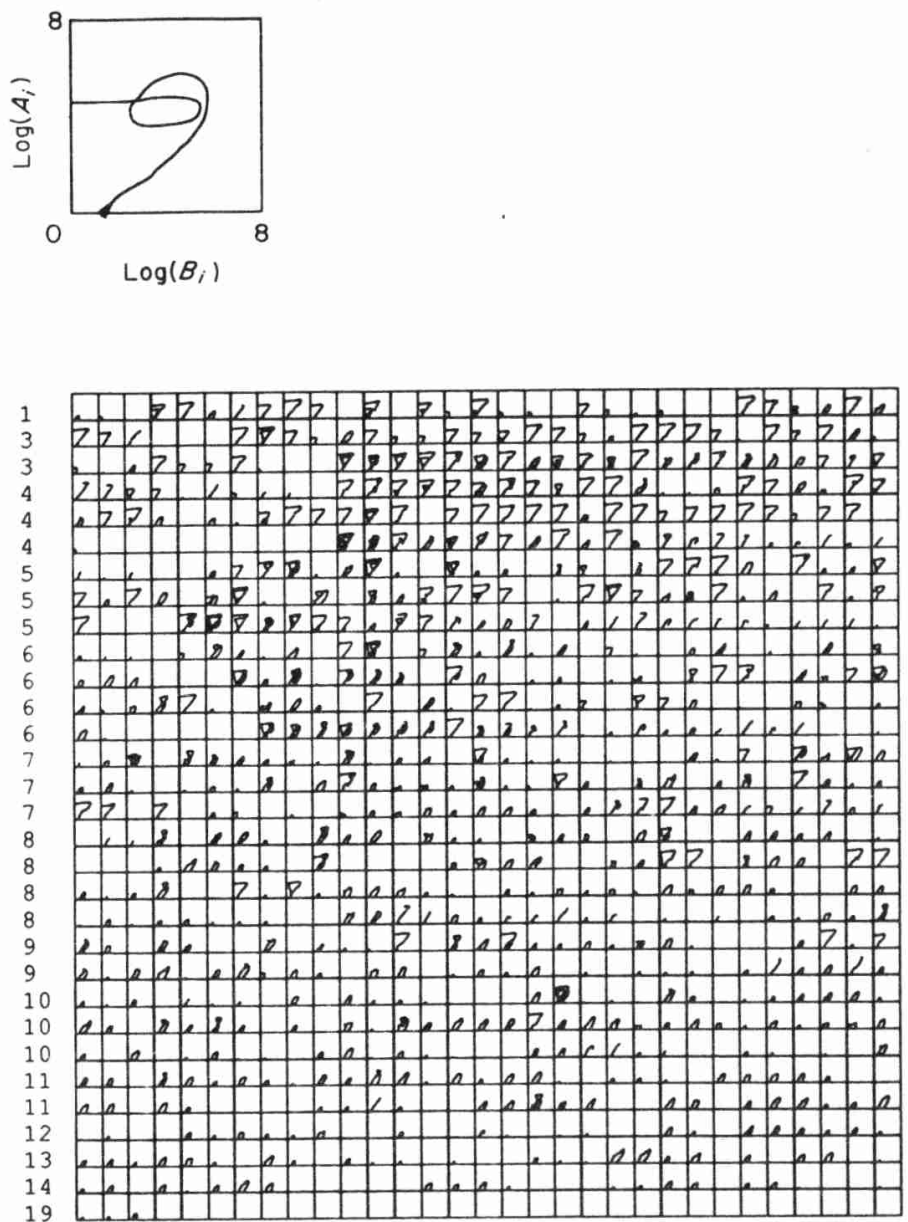

FIG. 5. The same dynamic behavior shown in Fig. 4 now plotted as $A_{i}$ vs. $B_{i}$. The vertical antibody and the horizontal $B$ cell axes are logarithmic (base 10) and run from 1 to $10^{8}$. Clones are again sorted by connectivity. Since the number of columns differs from that in Fig. 4, rows start differently.

been assumed that the antibody concentration follows the B cell population level, and thus a single variable is used to represent both. In Fig. 5(a) proportionality between antibody and B cells would be depicted by a diagonal line running from the lower left to the upper right, i.e. the antibody concentration would be low when the B cell population was small, and high when the B cell popolation was large. As one can see this is not necessarily the case. Antibodies and B cells have different lifetimes and thus need to be considered separately. In fact, this difference in lifetimes seems to be essential in generating the complex dynamical behavior characteristic of the two clone model (Fig. 3).

Figures 4 and 5 illustrate several other things. The behavior of most clones is typically oscillatory. Scanning from the top to the bottom one observes that the 
highly connected antibodies, i.e. those at the bottom of the figures, are short-lived and occur only during early life. Plotting the lifetime of the clones vs. their connectivity, one finds an inverse relation (not shown). Highly connected clones tend to be suppressed (i.e. they are over-stimulated); they can therefore only be maintained during early life when all clones are still small and the network has not yet filled with antibodies [see Fig. 6(b)]. Clones that maintain themselves during many oscillatory cycles dominate at low connectivity values (i.e. in the top third of Figs 4 and 5). Clones with a long lifespan go through several rounds of oscillation. A large number of clones display the "quiescent" behavior defined in Fig. 2. Quiescent clones in Fig. 4 have a region in which they form a straight diagonal line (e.g. plots 4 and 5); phase plots of the same quiescent clones in Fig. 5 end with a nearly horizontal trajectory that hits the $y$-axis (where the B cells die out). In these large networks, the large majority of the quiescent clones disappear following their quiescent period. During the quiescent period the B cells of these clones fail to proliferate. Thus, due to the absence of a supply from the bone marrow, $m=0$, they die out rapidly. The final removal of the idiotpyic antibody is usually rapid because once the idiotype concentration falls low enough to become stimulatory again, complementary B cells start to produce anti-idiotypic antibodies that hasten the removal of idiotypic antibody by complex formation.

Figure 6 shows the variation in time of some global characteristics of this example network for several values of the clonal production rate (i.e. $M=10,20,40$; lines

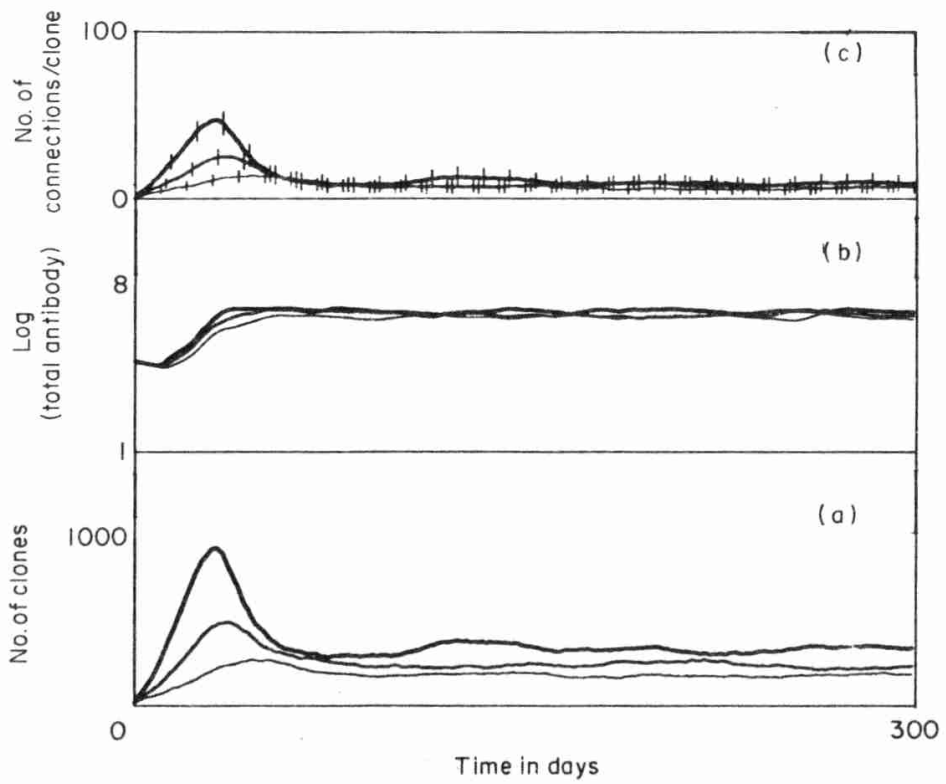

FiG. 6. Time dependent characteristics of the $d_{C}=10^{-3}, \theta_{2}=10^{4}, T=8, P($ match $)=0.05$ network displayed in Fig. 4. We show three values of bone marrow production: $M=10, M=20$, and $M=40$. Lines increase in thickness with $M$. (a) The number of clones in the network, (b) the total antibody on a logarithmic scale (base 10), and (c) the average connectivity (the bars indicate 1 S.D.). 
increase in thickness with $M$ ). The size of the network measured in terms of the number of clones [Fig. 6(a)], has a large peak during the first month, whose height increases with $M$. This early peak sharply declines by the third month and the network attains an equilibrium size around which it fluctuates. The total amount of antibody produced by the network is shown in Fig. 6(b). Starting with a slow decline of the maternal antibodies, the total antibody concentration increases until a steadystate level of about $3 \times 10^{6}$ units is attained. The daily average number of connections per clone, i.e. the connectivity of the network is shown in Fig. 6(c). As in Fig. 6(a), Fig. 6(c) shows an early peak and attains an equilibrium around day 100. This equilibrium is about six to nine connections/clone.

The fact that equilibrium levels are attained in Fig. 6 shows that the idiotypic network has certain self-structuring properties: the network selects a certain size, connectivity and antibody level. Coutinho has claimed that the immune system can "count". Our model is in some sense a verification of this principle, since once the network fills with clones it ceases to grow. The stable properties are surprising since each individual member of the network oscillates, and because the individual members are continuously being renewed. These self-structuring properties are further investigated below by analyzing a series of networks in which $P$ (match) is varied

\section{COVERING THE SHAPE SPACE}

Bit-strings of length $L=32$ are points in a binary "shape space" of 32 dimensions, i.e. the $32-\mathrm{D}$ hypercube. Because we only require a complementary match over part of each bit-string (i.e. $T<L$ ) each individual bit-string can match a set of complementary strings and hence will cover a relatively large part of the shape space. In fact, the proportion of the shape space covered by each individual random string is equal to $P$ (match). Therefore, the proportion $S$ of shape space that is covered by a random collection of $n$ strings is

$$
S=1-[1-P(\text { match })]^{n} .
$$

Thus, the number of strings that is required for covering the proportion $S$ of the shape space is

$$
n_{S}=\frac{\log (1-S)}{\log (1-P(\text { match })} .
$$

We will generally use a $99 \%$ coverage of the shape space (i.e. $S=0.99$ ) as an initial prediction of how large the network would grow if it were filling the shape space. We will call this estimate based upon eqn (6), $n_{99}$. Although this estimate-which is based upon random strings-will prove to be useful, we will also show that the shape space is not filled randomly at all. Shape space coverage is an interesting approach because it corresponds to the immunological concept of "repertoire completeness". A complete repertoire means full coverage of shape space. 
Expanding eqn (6) in a Taylor series

$$
n_{S}=\frac{-\log (1-S)}{P(\text { match })}+\frac{\log (1-S)}{2}+\frac{\log (1-S) P(\text { match })}{12}+\cdots
$$

For small values of $P($ match $), n \simeq-\log (1-S) / P($ match $)$. For $S=0.99, n \simeq$ $4 \cdot 6 / P($ match $)-2 \cdot 3+\cdots$. In our simulations $P($ match $) \leq 0 \cdot 21$. Consequently, there is at most $10 \%$ error if we assume $n=4 \cdot 6 / P$ (match). Thus, to a good approximation we find that to cover the shape space with random antibodies requires a number of antibodies that varies inversely with $P($ match $)$, i.e. $n \sim 1 / P($ match $)$.

\section{ANTIBODY MATCHING PROBABILITY}

The three self-structuring properties of the network are plotted, in Fig. 7, as a function of $P$ (match) for three different values of the bone marrow supply rate $M$. The heavy line in Fig. 7 (a) depicts $n_{99}$, the number of clones required for filling $99 \%$ of the shape space. The observed values are generally larger than $n_{99}$. The match probabilities range from $0 \cdot 005-0 \cdot 205$; the actual matching parameters were varied from $T=11$ to $T=6$ (for $L=32$ ). The equilibrium size of the network strongly depends on $P$ (match) [Fig. 7(a)]. Networks comprised of sticky receptors [e.g. $P($ match $)>0 \cdot 1]$ remain very small, i.e. $<200$ clones. Conversely, whenever receptors are specific [e.g. $P($ match $)<0 \cdot 01$ ], the networks become very large. The shape of the observed curves is similar to that of the $n_{99}$ curve. The number of clones is thus inversely related to $P$ (match), and we conclude that our model networks can only be large if idiotypic interactions are highly specific. Systems with highly specific receptors will generate very large networks.

Networks comprised of clones with highly specific receptors not only consist of more clones, than do networks of less specific clones, they also contain more antibody [Fig. 7(b)]. This panel looks very much the same as Fig. 7(a), i.e. cells with sticky receptors form small networks, whereas cells with specific receptors form large networks containing large amounts of total antibody. The average size of the individual antibody populations $A_{i}$ is largely independent of $P$ (match) [Fig. $\left.7(\mathrm{~d})\right]$.

For sufficiently large values of $P$ (match) the connectivity, i.e. the average number of connections per clone, becomes independent of $P$ (match) [the thin lines in Fig. 7 (c) ]. For low values of $P$ (match) the connectivity increases with $P$ (match) until it saturates at about ten connections clone. Figure $7(\mathrm{c})$ illustrates that one cannot deduce receptor stickiness from the connectivity of the network. If one calculates the "expected connectivity" of a network, i.e. $P$ (match) multiplied by the average number of clones in the network [as provided in Fig. 7(a)], one obtains the heavy lines in Fig. 7(c). This shows that, although sticky networks are comprised of few clones, one would expect more connections per clone on the basis of the binding properties of the receptors. Even for the highly specific networks, the expected connectivity is higher than the actual connectivity. Thus, whatever $P$ (match), these networks are selecting for clones with a low connectivity.

The early peak in the size of the network, seen in Fig. 6(a), depends on $P$ (match). If receptors are highly specific few newly generated clones match to the network; 

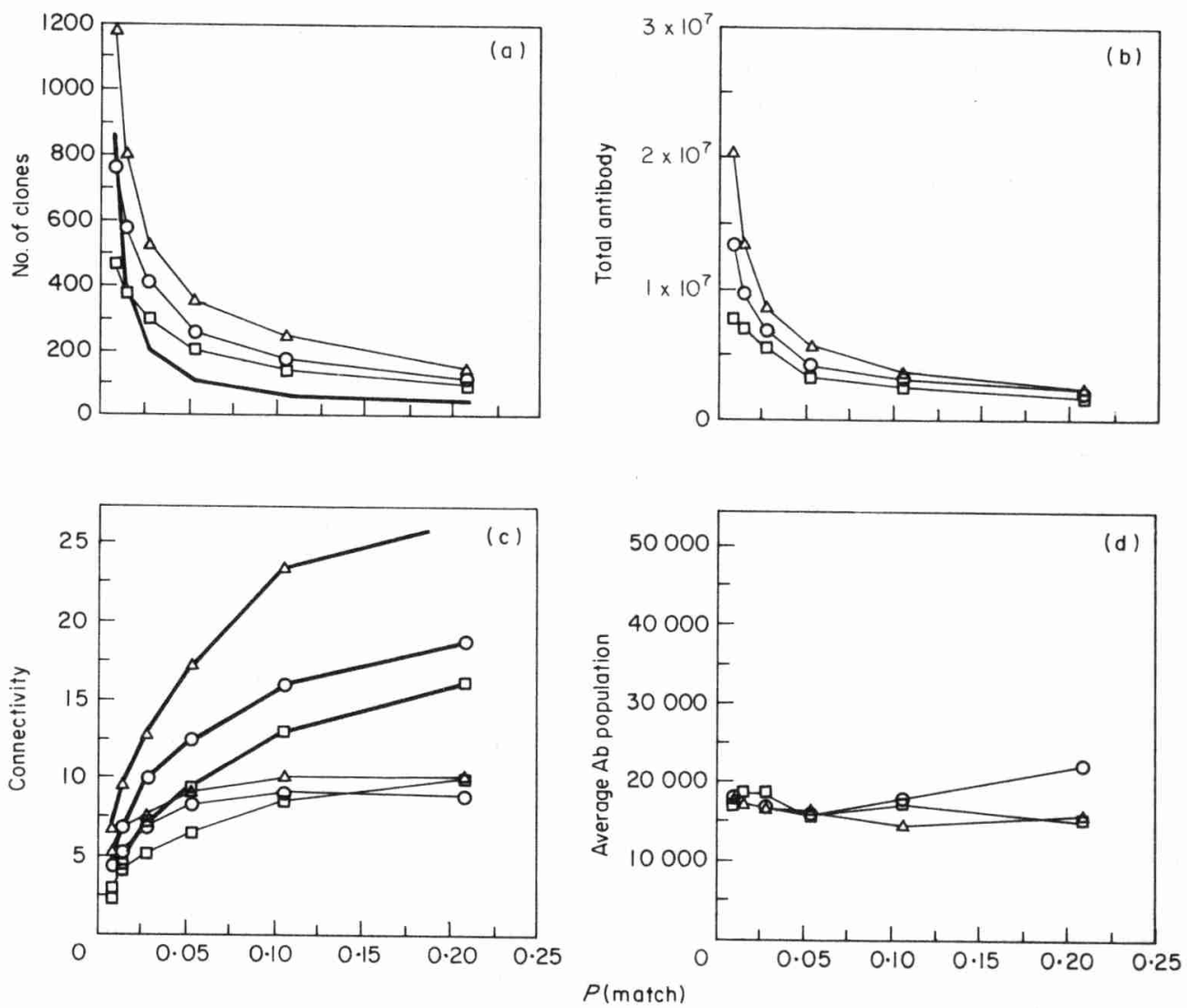

FIG. 7. Equilibrium characteristics of a series of networks varying $P$ (match) for $d_{C}=10^{-3}$ and $\theta_{2}=10^{4}$. The equilibrium values were determined by averaging over the last 100 days of a dynamic simulation. We show three values of $M: M=10$ : ( $\square$ ), $M=20:(\bigcirc), M=40:(\triangle)$. (a) The total number of clones (light lines), and the $n_{99}$ (heavy line). (b) The total antibody concentration. (c) The network connectivity (light lines), and the expected network connectivity (heavy lines). (d) The average antibody population.

as a consequence the number of clones increases slowly and the peak remains absent (not shown).

\section{AVERAGE FIELD}

Individual B cell populations proliferate best when their field, $\bar{A}_{i}$, is between $\theta_{1}$ and $\theta_{2}$. We therefore estimated the average field by calculating $1 / n \sum_{i=1}^{n} \bar{A}_{i}$. These average field values are slightly larger than $\theta_{2}$ and are largely independent of $P$ (match). There is a simple reason why the observed average field has to be close to $\theta_{2}$. If the fields were to be equally divided over the two equilibrium values $\theta_{1}$ and $\theta_{2}$, the average field would be $\left(\theta_{1}+\theta_{2}\right) / 2$, which roughly equals $\theta_{2} / 2$ because 
$\theta_{2} \gg \theta_{1}$. The fact that the average field is slightly larger than $\theta_{2}$ implies that the majority of the clones in the network are suppressed (i.e. $\theta_{2}>\sqrt{\theta_{1} \theta_{2}}$ ). In the present networks typically $60 \%$ of the clones are suppressed (i.e. have a field larger than $\sqrt{\theta_{1} \theta_{2}}$ ), and $30 \%$ of all antibody populations are of a suppressive size (i.e. are larger than $\sqrt{\theta_{1} \theta_{2}}$ ). Both percentages are largely independent of $P$ (match) (not shown).

\section{MOST CLONES ARE SUPPRESSED}

In his original formulation of the network hypothesis Jerne (1974) suggested that the dominant interactions in networks are suppressive, so that in the absence of antigen, clones normally are suppressed. This view is supported by our model even though we have no explicit suppressive interactions between clones. If receptors are highly specific, as we expect to be the case in real immune systems, each clone will be connected to relatively few others. In this situation, our simulations show that many clones can be incorporated into the network before the connectivity increases to the point that the average field becomes suppressive. In principle, networks could also attain an equilibrium size with the average field at $\theta_{2}$ by evolving into a system with few but highly connected clones. Interestingly, this does not occur. Rather our networks select for low connectivity. This can be explained by the "dominance" of specificity. When specific clones compete with multireactive clones for presence in the network, the specific clones tend to win because multireactive clones are more prone to be suppressed (i.e. their larger field more easily exceeds $\sqrt{\theta_{1} \theta_{2}}$ ). Thus our simultions show that network interactions can influence the expressed repertoire in an animal and over time select for clones with few connections. Thus even though neonatal immune systems are dominated by clones which secrete highly multireactive antibodies, in this model they are slowly replaced by clones expressing more specific receptors that react with fewer other members in the network. This is in conformity with what it seen during the development of the immune system (Holmberg et al., 1986b).

\section{ANTIBODY REMOVAL BY COMPLEX FORMATION}

A very important parameter in our model is $d_{C}$, the rate of removal of antibody due to complex formation. Little is known about the in vivo formation of complexes between idiotypic and anti-idiotypic antibodies. Here we have only considered complexes made out of two antibodies. This is certainly too simple because ten-valent IgM antibodies can form a spectrum of complexes differing in size and differing in the ratio of idiotypic and anti-idiotypic antibodies (cf. Stockmeyer, 1943; Goldberg, 1952). Moreover, the rate of removal of the complexes may depend on their size, further complicating the development of realistic models of antibody removal. Rather than deal with these issues directly, we can get some idea of the sensitivity of our results to our simplifying assumptions by varying the parameter $d_{C}$. This parameter is a crucial bifurcation parameter in the two-clone models (Fig. 3 and De Boer et 
al., 1990) and thus we anticipate that the results presented above with $d_{C}=10^{-3}$ may depend on this particular choice.

In Fig. 8 we plot the variation of $\boldsymbol{A}_{i}$ with time for all of the clones in a network in which $d_{C}$ is increased ten-fold. As expected, this increase influences the network behavior. Comparing with Fig. 4 we see that the networks consist of more clones (1135 in Fig. 9 vs. 933 in Fig. 4), oscillations are more rapid, and clones persist during prolonged oscillations. Quiescent behavior occurs only seldomly. Quiescent behavior is also uncommon for larger and smaller values of $P$ (match) (not shown). Again, scanning from the top to the bottom, the most interesting behavior is seen in the top half of the figure. The highly connected clones depicted at the bottom of
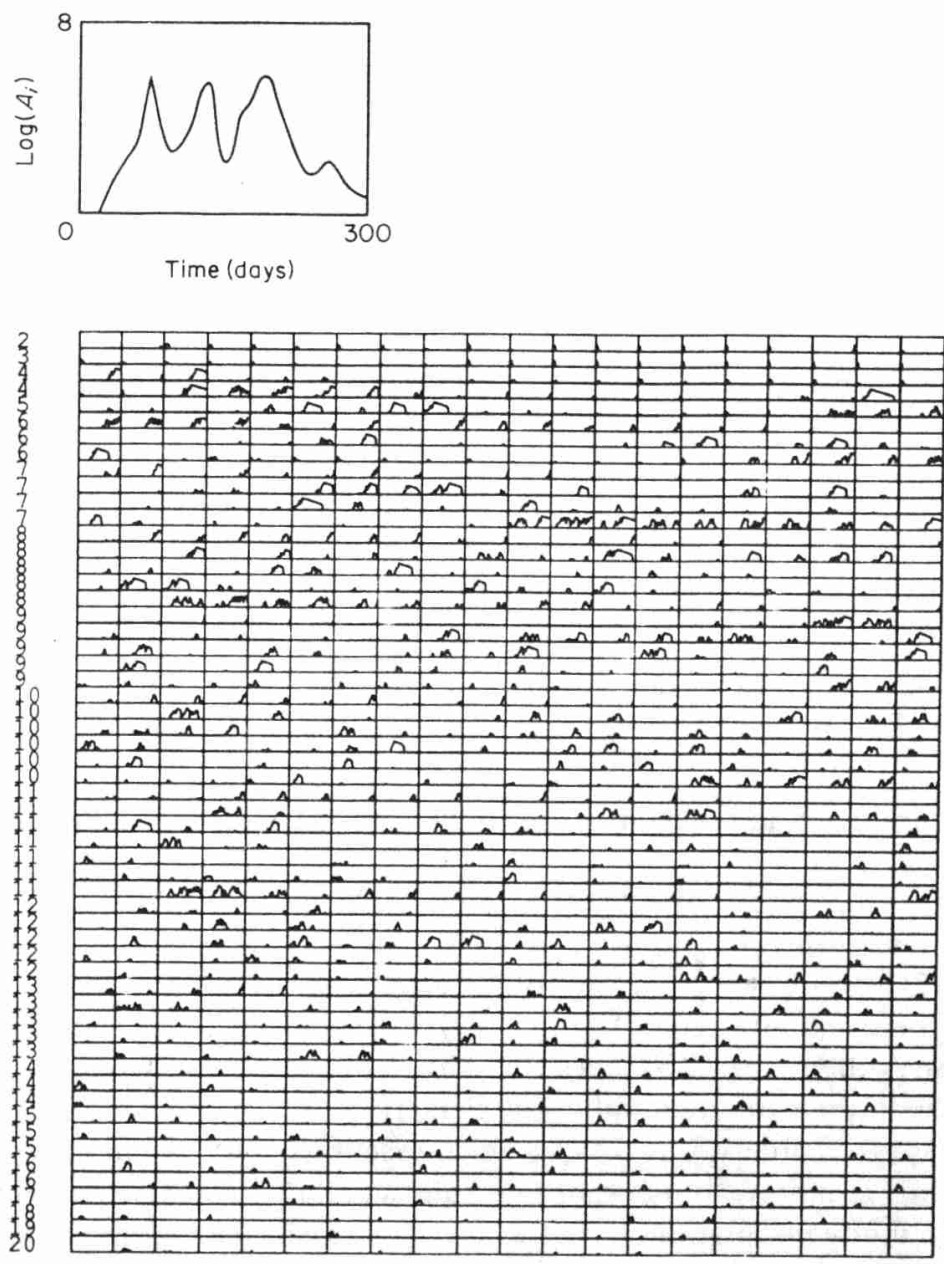

FIG. 8. The behavior of a network with a ten-fold increase in the rate of complex formation $d_{C}=0 \cdot 01$ and $\theta_{2}=10^{4}, T=8, P($ match $)=0 \cdot 05$. The network contained 1135 clones. We plot $\log A_{i}$ vs time. Clones are sorted by their connectivity as in Fig. 4. 

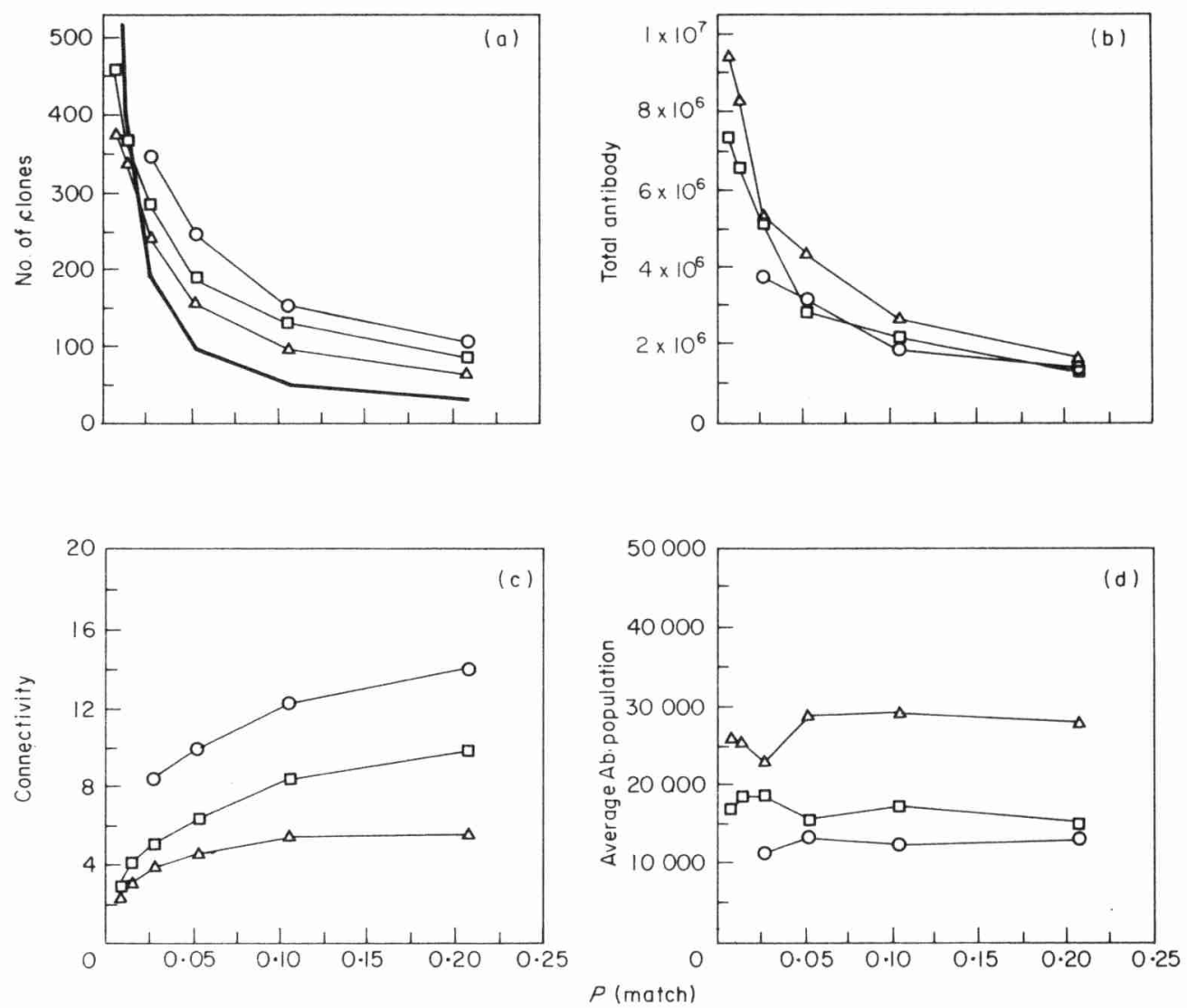

FIG. 9. The dependence of the equilibrium characteristics of the networks on $P$ (match), for $M=10$, $\theta_{2}=10^{4}$ and three values of $d_{C}$. We use the following symbols: $(\triangle): d_{C}=10^{-4},(\square): d_{C}=10^{-3},(\bigcirc)$ : $d_{C}=10^{-2}$. (a) The total number of clones (light lines), and the $n_{99}$ (heavy line). (b) The total antibody concentration (c) The network connectivity. (d) The average antibody population.

the figure are again unsuccessful, and only occur early in the simulation when the network is small.

In Fig. 9 we compare, for $M=10$, the equilibrium properties obtained in three series of simulations with $d_{C}=10^{-3}$, our standard value $(\square), d_{C}=10^{-2}$, a ten-fold increase $(O)$ or $d_{C}=10^{-4}$, a ten-fold decrease $(\triangle)$. We see that increasing $d_{C}$ increases the size of the network [Fig. 9(a)], hardly affects total antibody [Fig. 9(b)], and increases the network connectivity [Fig. 9(c)]. With $d_{C}$ increased ten-fold, the networks with their different values of $P$ (match) on average consists of $25 \%$ more clones, $5 \%$ less antibody, and $52 \%$ more connections. Because antibody is removed more rapidly antibody populations remain smaller. Figure $9(\mathrm{~d})$ shows that the average antibody population decreases if $d_{C}$ increases. Therefore, a higher average connectance is required to produce the same field. The model network thus incorporates more clones. The average field value is largely independent of $\boldsymbol{P}$ (match) and $d_{C}$ (not shown). 
THE ONSET OF SUPPRESSION $\boldsymbol{\theta}_{2}$

Another parameter that is expected to affect competition among clones is $\theta_{2}$, the parameter determining when field values start to become suppressive (i.e. larger than $\sqrt{\theta_{1} \theta_{2}}$ ). In Fig. 10 we compare, for $M=10$, the equilibrium properties of networks for three values of $\theta_{2}: 10^{3}, 10^{4}$, and $10^{5}$. The dynamic behavior of networks in which $\theta_{2}=10^{5}$ (not shown) is comparable to that displayed in Fig. 4 for $\theta_{2}=10^{4}$ in the sense that quiescent behavior is common, and the rate of oscillations seems similar. The networks with $\theta_{2}=10^{3}$ behave differently. Clones tend to be long-lived, the amplitude of the oscillations is smaller, and the antibody and B cell population tend to vary proportionally. Figure 10 (b) and (d) show that antibody populations tend to vary in proportion to $\theta_{2}$. In general, both the number of clones [Fig. 10(a)] and the connectivity [Fig. 10(c)] increase somewhat if $\theta_{2}$ is varied ten-fold in either direction from the standard value $\theta_{2}=10^{4}$. Total antibody levels [Fig. 10(b)], and
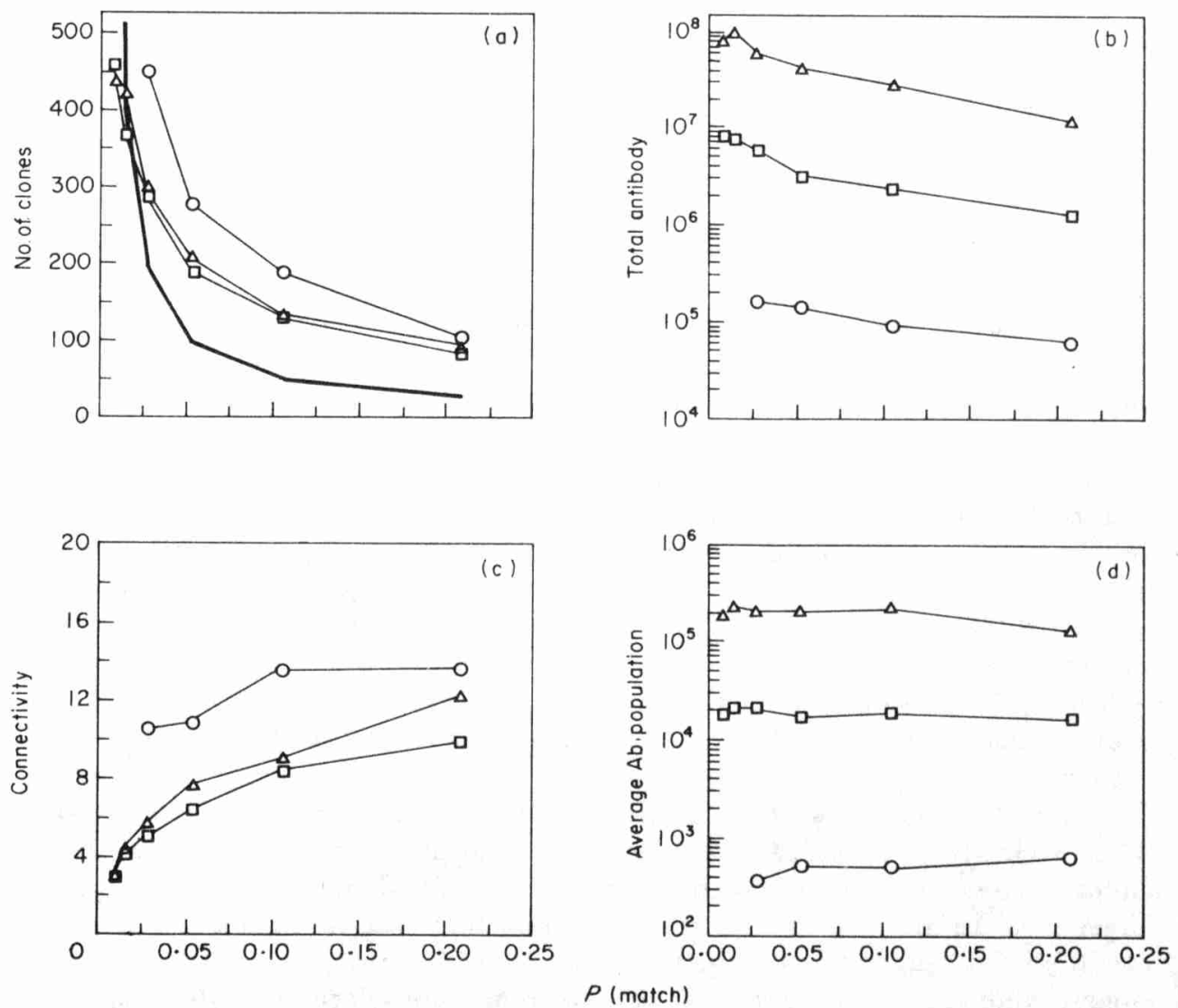

FIG. 10. The dependence of the equilibrium characteristics of the networks on $P$ (match), for $M=10$, $d_{c}=10^{-3}$ and three values of $\theta_{2}$. We use the following symbols: $(\bigcirc): \theta_{2}=10^{3},(\square): \theta_{2}=10^{4},(\triangle): \theta_{2}=10^{5}$. (a) The total number of clones (light lines), and the $n_{99}$ (heavy line). (b) The total antibody concentration.

(c) The network connectivity. (d) The average antibody population. 
the average antibody population [Fig. 10(d)], change about ten-fold upon a ten-fold change of $\theta_{2}$. The average field is largely independent of $P$ (match), but also changes ten-fold upon a ten-fold change of $\theta_{2}$ (not shown).

\section{The Size of Idiotypic Networks}

BONE MARROW SUPPLY RATE

Figures 7,9 and 10 show the size and connectivity of the network for three values of the rate at which novel clones are supplied from the bone marrow: $M=10,20$, 40. Biological networks however, have a far larger rate of supply of clones from the bone marrow. The bone marrow of an adult mouse produces about $5 \times 10^{7}$ pre- $\mathrm{B}$ cells/day (Opstelten \& Osmond, 1983). Since these pre-B cells divide, the production should definitely be less than $5 \times 10^{7}$ different specificities. We think $M=10^{5}-10^{6}$ as a reasonable range. Here we study the effect of increasing $M$ in our network with the most multireactive antibodies [i.e. $P($ match $)=0 \cdot 205]$. [Note that a connectivity of about $20 \%$ has been reported in newborn mice (Holmberg et al., 1984; Kearney et al., 1987); but as we have argued above, due to selection such connectivity estimates need not correspond to the actual $P$ (match).] In Fig. 11, we analyze the average size, the total antibody concentrations, and the connectivity of these networks as a function of $M$.

The time to attain an equilibrium size depends on $M$. Therefore the dynamic simulations, whose results are summarized in Fig. 11, were carried out for 600 instead of 300 days. Simulations of networks with large $M$ values take over a day to simulate on a SUN Sparcstation 1. Because much of the simulation time is spent on calculating the early peak, which involves thousands of clones if $M$ is large, we attempted to start our simulations near equilibrium. At day zero we filled the networks with clones until the average field of each clone was larger than the preserved average field value of $\theta_{2}$. The respective clones sizes were distributed around $\theta_{1}$ or $\theta_{2}$. From our earlier simulations we know that approximately $30 \%$ of the clones are larger than $\sqrt{\theta_{1} \theta_{2}}$. Thus the size of $30 \%$ of the clones was set to a random value around $\theta_{2}$ and $70 \%$ of the sizes to a value around $\theta_{1}$ (using $10 \%$ standard deviation). Following this filling procedure, networks were simulated for 600 days. We performed both a linear and a logarithmic fitting procedure to the data. In each panel of Fig. 11 we present the fit that yielded the highest correlation coefficient.

Figure 11(a) shows that the size of the network tends to increase linearly with $M$. It is clearly of biological interest to be able to predict the size of networks in animals. To do so we need to extrapolate to values of $M$ two orders of magnitude larger than we are now able to attain in simulation. The linear fits in Fig. 11(a) would however suggest that biological networks, with $M \simeq 10^{6}$, consist of order $10^{6}$ clones. Note that, if this is indeed the case, repertoire selection is strong because $10^{6}$ clones would need to be neglected on a daily basis. We have tested the robustness of these results by a reconsideration of what we mean by the size of the network. In our model the network is not static, new clones join the network and unsuccessful 

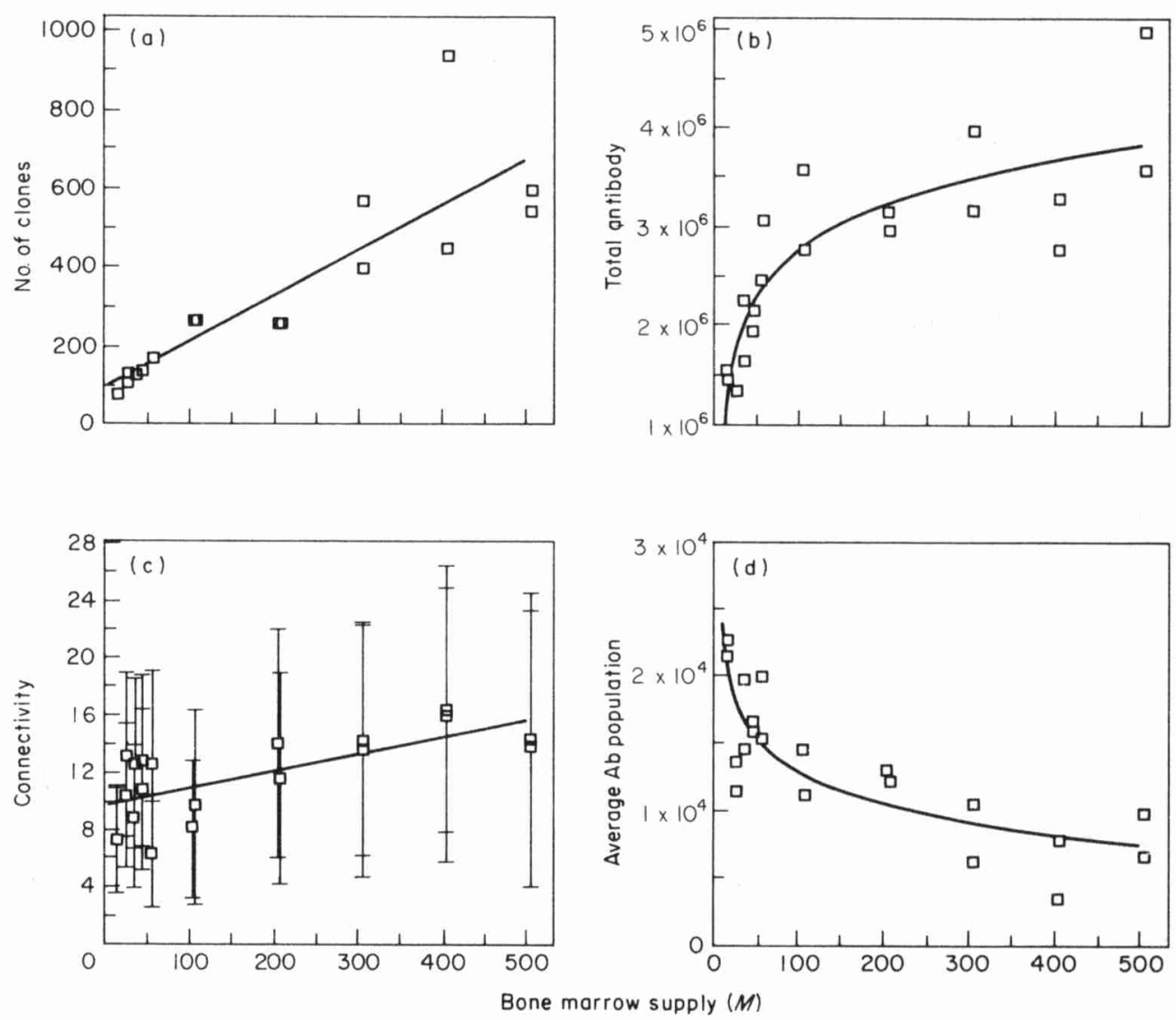

FiG. 11. The size of the network as a function of $M$ for $d_{C}=10^{-3}, \theta_{2}=10^{4}, T=6, P($ match $)=0 \cdot 205$. The averages were determined over the last 300 days of simulations lasting 600 days. Depending on the respective correlation coefficients we either show a linear or a logarithmic fit. (a) The number of clones in the network as a function of $M$. (b) Total antibody. (c) The average connectivity. (d) The average antibody population. In (c) the mean connectivity plus or minus 1 S.D. is indicated for each simulation run.

clones are eliminated from the network. Only some clones are able to maintain themselves in the network. These clones might constitute a "core" of the network. Thus, we recalculated the data on the size of the network, considering only clones that were present for more than 1 week. This did not change our estimate of the network size qualitatively, which suggests that our simplier measure of network size suffices.

The connectivity of the networks, the observable which turned out to be fairly independent of $P$ (match), also seems to be fairly independent of $M$. Figure 11(c) suggests a characteristic immune network connectivity of about ten to 15 connections/clone, and shows a slow linear increase with $M$. Note however, that the average connectivity has large standard deviations, making it difficult to estimate the average connectivity's dependence on $M$. Additional work needs to be carried out in order 
to establish whether the same characteristic connectivity applies for networks with different $P$ (match). The total amount of antibody in the network also increases with $M$ [see Fig. 11(b)]. An extrapolation of the logarithmetic fit suggests however that the increase in total amount of antibody would become negligible if $M$ were to increase to the biological estimate of $M \simeq 10^{6}$. The slow increase in total antibody is in agreement with the decrease in the average size of the antibody populations when $M$ increases [Fig. 11(d)]. The average field experienced by each clone also decreases slightly if $M$ increases (not shown). The range of variation in the average field is small however. Additionally, the average field always remains suppressive for the range of $M$ values that we are presently able to investigate.

\section{HAMMING DISTANCES IN THE SHAPE SPACE}

The similarity in shape between the observed curves and the $n_{99}$ curve shown in Figs 7, 9, and 10 suggests that the observed equilibrium size of a network is related to the number of clones needed to cover the shape space. If this is true, it then becomes puzzling why increasing $M$ increases the equilibrium size (Figs 7 and 11). Once the shape space has filled up, introducing novel clones should not give rise

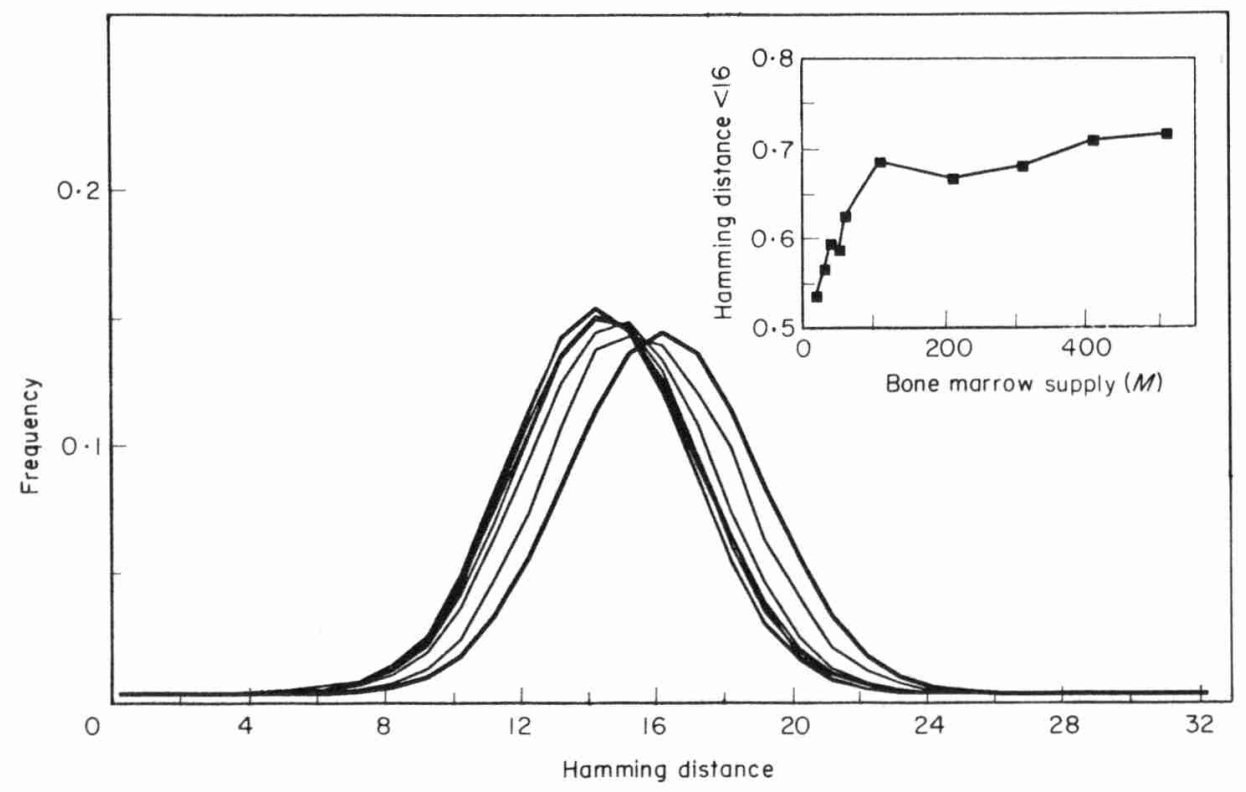

FIG. 12. Hamming distances in the shape space. For a subset of the 20 networks depicted in Fig. 11, we computed the frequency distribution of Hamming distances between all pairs of clones. Samples were taken at 30 day intervals between day 300 and 600 , and the frequency distribution of the total of the samples is plotted. The frequency distributions are indicated by the light lines in the figure. From right to left the distributions correspond to $M=10,50,100,200,300,400,500$. The distribution with its maximum at 16 , indicated by a heavy line, depicts the expected binomial distribution. The inset shows for the same subset of networks the proportion of the distribution that lays left of the expected maximum of 16 bits. 
to a larger network. We analyze this problem in two ways. First, we will study Hamming distance in shape space. Second, we will present a phenomenological equation describing the number of clones in the network.

For a representative subset of the 20 networks in Fig. 11, we have scored the Hamming distances between all possible pairs of bit-strings. The Hamming distances were sampled at 30-day intervals between day 300 and day 600 . The frequency distributions of the respective Hamming distances in the total of 11 samples of each network is plotted in Fig. 12. The expected distribution for a randomly filled shape space is a binomial distribution with its maximum at $L / 2=16$ different bits. The binomial distribution is depicted by the heavy line in the figure. The observed distributions all lie to the left of the expected distribution. Thus, the bit-strings found in the network are more similar than expected. Furthermore, increasing $M$ increases the deviation from the expected distribution. The same result is summarized in the inset of Fig. 12 which depicts the proportion of the observed distribution that lies left of the expected maximum $L / 2=16$. We conclude that clones tend to become more similar if $M$ increases. Because similar bit-strings will overlap in the region of the shape space that they cover, more clones can be packed into the same space.

Because connections are based upon complementary matches, the tendency of clones to be similar reduces the probability that clones match, i.e. reduces the connectivity of the network. The observation that the connectivity always remains lower than expected, which is the basis for our conclusion that the networks select for clones with few connections, can be explained by these results. Clones that are similar to an established (i.e. successful) clone have a two-fold selective advantage. First, they will share the successful field value, and second they will not be removed by complex formation with the successful antibody.

\section{A Phenomenological Model}

The equilibrium size that each network attains is not only determined by the filling of shape space but is also influenced by the dynamical parameters. Figures 9 and 10 , for instance, show that size of the network also depends on $d_{C}$ and $\theta_{2}$. Here we will propose a phenomenological equation for the number of clones, $n$, in the network. The number of clones being determined by the influx from the bone marrow (i.e. $M$ ), and their removal from the network. An early attempt to formulate a similar phenomenological model for the number of clones in the immune pepertoire was performed by Adam (1978). His approach differs from ours because he assumes (1) that idiotypes emerge by somatic mutation from established cells, and (2) that idiotypic interactions are suppressive.

The influx from the bone marrow is not really a constant because we have applied two constraints for incorporating clones into the network. Once the networks have attained equilibrium however, both constraints play hardly any role in the network dynamics. The first constraint that clones connect to the network is likely to be fulfilled because we have seen that the network covers nearly (i.e. $>99 \%$ ) the entire shape space. The second constraint was only a short-cut that should not affect the dynamics at all. Thus, we will treat the influx as constant. 
Clones are removed from the network if their degree of network stimulation, $\bar{A}_{i}$, is either too small or too large. Since clones sizes are roughly equal for all networks [Figs $7(\mathrm{~d}), 9(\mathrm{~d})$, and $10(\mathrm{~d})$ ], $\bar{A}_{i}$ will mainly depend on a clone's connectivity. For simplicity, we will assume here that the degree of clonal stimulation depends on the connectivity only. An estimate for the connectivity is the expected connectivity $C=n P$ (match). Note however, that this estimate is actually a rather poor one [Figs $7(\mathrm{c})$ and $11(\mathrm{c})$ ]. Due to the bell-shaped nature of the activation function [eqn (1)] clones will tend to be removed whenever the connectivity is too low or too high. A natural way of modeling this is to use the bell-shaped function

$$
f(C)=\frac{C^{\eta}}{\theta_{1}^{\eta}+C^{\eta}} \frac{\theta_{2}^{\eta}}{\theta_{2}^{\eta}+C^{\eta}},
$$

where $C=n P$ (match) and $\theta_{2}>\theta_{1}$. The window set by $\theta_{1}$ and $\theta_{2}$ is the connectivity range within which the degree of stimulation tends to prevent clonal removal from the network.

Thus, we propose as a phenomenological equation for $n$, the number of clones in the network,

$$
\frac{\mathrm{d} n}{\mathrm{~d} t}=M-d[1-f(C)] n
$$

where $M$ is the rate of supply of novel clones, $d$ is a parameter specifying the rate at which unsuccessful clones are removed, and $1-f(C)$ is the fraction of clones not stimulated to proliferate. We will derive below that $d \simeq 1$. The values of $\theta_{1}$ and $\theta_{2}$ can be estimated from our observation that the connectivity of the network generally varies between five to 15 connections/clone. We thus set $\theta_{1}=5$ and $\theta_{2}=15$. Having the thresholds so close, one needs to adjust the steepness of the function in order to obtain significant suppression in the region $C>\theta_{2}$. In Fig. 13(a) we plot eqn (7) for $\eta=1$ and $\eta=5$. A sigmoid function (i.e. $\eta>1$ ) seems most reasonable. For algebraic constraints, however, we set $\eta=1$ in the following analytic derivation.

Our biological estimates for the bone marrow production vary around $\boldsymbol{M}=$ $10^{5}-10^{6}$ clones/day. In our simulations however, we were unable to go beyond $M=500$ [see Fig. 11(a)]. In a large network with a high connectivity, i.e. $C \gg \theta_{2}$, we can approximate eqn (7) by its descending part $f(C) \simeq \theta_{2} /\left(\theta_{2}+C\right)$. Here we will derive an approximation for the size of the network $n$ as a function of large values of $M$. Solving eqn (8) for $\bar{n}$, the equilibrium value of $n$, then gives only one positive root

$$
\bar{n}=\frac{M}{2 d}\left(1+\sqrt{1+\frac{4 \theta_{2} d}{M P}}\right),
$$

where $P=P$ (match). For $M \rightarrow \infty$, this solution can be approximated by

$$
\bar{n}=\frac{M}{d}+\frac{\theta_{2}}{P}-\frac{\theta_{2}^{2} d}{P^{2} M}+\cdots .
$$


If we compare this with the observed values for $100 \leq M \leq 500$ in Fig. 11(a), we can estimate the order of magnitude of the respective terms in eqn (9). Since the turnover of clones $d$ is of the order of one to several days, $100 \leq M \leq 500, \theta_{2}=15$, and $P=0 \cdot 205$, the first term is large (i.e. of order rate $M$ ), the second smaller (i.e. of order $\theta_{2}$ ), and the third term is the smallest. Thus we can approximate eqn (9) by $\bar{n}=M / d$. The observation that for large $M$ the number of clones scales linearly with $M$ [Fig. 11(a)] is thus in confirmity with our phenomenological model. Because the observed relationship in Fig. 11(a) is roughly $\bar{n}=M$, we set $d=1$.

In Fig. 13(b) we numerically check this analytic prediction by plotting $\bar{n}$ employing the full eqn (7). The heavy line corresponds to $\eta=5$; for comparison we show the light line corresponding to $\eta=1$. For $M>100$ these curves approach the linear function $\bar{n}=M$, confirming our analytic approximation. For low values of $M$, the equilibrium size varies as either an S-shaped (for $\eta=5$ ), or curved (for $\eta=1$ ) function of $M$. Close inspection of the simulation results in Fig. 11(a) also reveals
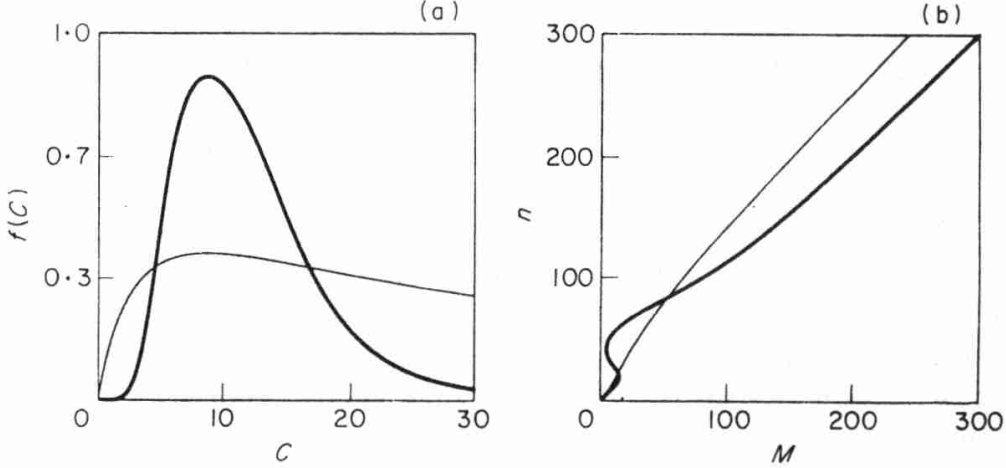

(c)

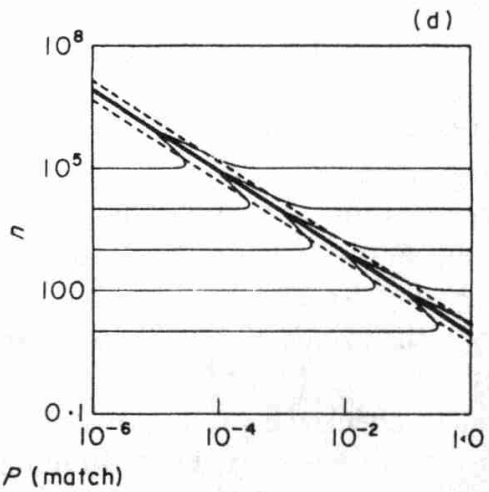

FIG. 13. The equilibrium value of $n$ of our phenomenological model. (a) The bell-shaped eqn (7) as a function of the connectivity $C$. The heavy line corresponds to $\eta=5$, the light line to $\eta=1$. (b) The equilibrium value of $n$ as a function of bone marrow production rate $M$. The heavy line corresponds to $\eta=5$, the light line to $\eta=1$. (c) The equilibrium value of $n$ as a function of $P$ (match) for $M=10,20$, and 40. The heavy black line denotes a connectivity of $C=\sqrt{\theta_{1} \theta_{2}}=8 \cdot 66$. The heavy dashed lines denote a connectivity of $C=\theta_{1}$ and $C=\theta_{2}$ respectively. (d) The equilibrium value of $n$ as a function of $P$ (match) for $M=10,100,10^{3}, 10^{4}$, and $10^{5}$. The heavy lines are equal to those in (c). 
a deviation from the linear relation $\bar{n}=M$. Due to the noise in these small networks however, the data are insufficient to distinguish between the $\mathrm{S}$-shaped and the curved relationship.

Both the space space analysis (i.e. $n_{99}$ ) and the simulation results show an inverse relationship between $n$ and $P$ (match). Here we numerically study the same relationship in the phenomenological model using the full form of $f(C)$, eqn (7) for $\eta=5$. In Fig. 13(c) the light lines depict $\bar{n}$, found by solving $\mathrm{d} n / \mathrm{d} t=0$, as a function of $P$ (match) for $M=10,20$ and 40 , respectively. The heavy black line represents the connectivity $C=\sqrt{\theta_{1} \theta_{2}}=8.66$, above it the connectivity is higher, below it is lower. The two heavy dashed lines represent $C=\theta_{1}$ and $C=\theta_{2}$, respectively. To the right of the line $C=\sqrt{\theta_{1} \theta_{2}}$, the $\bar{n}$ curves seem very similar to the observed curves in Figs 7 (a), 9 (a), and $10(\mathrm{a}) ; \bar{n}$ is roughly proportional to $1 / P$ (match). This result of the phenomenological model is in agreement with the outcome of the simulations and the filling of the shape space. Additionally, our phenomenological model suggests for $C>\sqrt{\theta_{1} \theta_{2}}$, that suppression should dominate in the network [see Fig. 13(a)]; this is exactly what we observed in our simulations. Conversely, to the left of the line $C=\sqrt{\theta_{1} \theta_{2}}, \bar{n}$ increases if $P$ (match) increases. Additionally, the $\bar{n}$ curves are $\mathrm{S}$-shaped (for $\eta=5$ ). For low values of $P$ (match) we thus find three equilibrium values. Two of them, i.e. the lower and the upper one, are stable. Thus, over the entire range of $P$ (match), eqn (7) predicts the existence of two equilibrium regimes that we call the "suppressive" and the "stimulatory" regime, respectively. For large values of $P$ (match) suppression dominates, and $\bar{n}$ decreases with $P$ (match). For low values of $P$ (match) stimulation dominates, and $\bar{n}$ increases with $P$ (match). For low values of $P$ (match) both types of equilibria might coexist.

As argued above, the equilibria in the suppressive regime correspond to the equilibria of the simulation model shown in Figs 7(a), 9(a), and 10(a). Here we discuss the other equilibria found in the phenomenological model. First, if the network connectivity $C$ is too low or too high, $f(C) \simeq 0$, and $\bar{n} \simeq M / d$. Immunologically speaking, this means that there is no network and that the number of clones is determined by the balance of source from bone marrow $M$ and turnover $d$. In Fig. 13(c) this is shown as the horizontal parts of the curves $\bar{n}$. Whenever $\bar{n}>M / d$ clones are maintained in the immune system due to network stimulation. In the stimulatory regime the phenomenological model has a low stable-state and an intermediate unstable-state. The low stable-state corresponds to $\bar{n}=M / d$ for most values of $P$ (match). However, close to the limit point where the stable and unstablestate merge, the graph of $\bar{n}$ vs. $P$ (match) is curved and some clones are maintained in the network. i.e. $\bar{n}>M / d$.

In order to check the predictions of the phenomenological model for low values of $P$ (match), we performed additional simulations for lower values of $P$ (match). We studied our standard $M=10, d_{C}=10^{-3}, \theta_{2}=10^{4}$ network for $T=11, P($ match $)=$ 0.005 , and $T=12, P($ match $)=0.003$ for different initial conditions. Starting with few clones, these networks all attained an equilibrium with few clones $(<40)$ and a low connectivity (about 1 ). This is in agreement with the stimulatory equilibria close to the lower limit point of the phenomenological model. Approximately $30 \%$ of the clones are suppressed in these equilibria. Starting with many clones, the 
networks attained the typical suppressive equilibria displayed in Figs 7(a), 9(a), and 10(a). Starting at intermediate numbers of clones, the dynamics were slow, suggesting the presence of the unstable equilibrium from which the system is escaping. After long transients the networks settled in either the lower or the upper stable equilibrium. A closer inspection of the connectivity curves in Fig. 7 also suggest that the connectivity starts to decrease at low values of $P$ (match). We now conclude that these are on the verge of switching to the lower equilibrium.

In an attempt to extrapolate to highly specific interactions [e.g. $P($ match $)=10^{-5}$ ] and a high bone marrow influx (e.g. $M=10^{5}$ ) we plot the $\bar{n}$ curve on logarithmic scales in Fig. 13(d). The various light lines correspond to $M=10,100, \ldots, 10^{5}$. The heavy lines again correspond to $C=\theta_{1}, \sqrt{\theta_{1} \theta_{2}}$, and $\theta_{2}$. This shows that for any value of bone marrow production, there will be a region of specificities in which both the stimulatory and the suppressive equilibria exist.

In our simulations we observed that the connectivity was lower than expected, and was relatively independent of $P$ (match). In the phenomenological model the connectivity was made equal to the expected connectivity. However, in Fig. 13(c) and (d) one can see that within the interesting range of specificities, where the network maintains clones in the system (i.e. where $\bar{n}>M / d$ ), the $n^{\prime}=0$ solutions lie in the region $\theta_{1}<C \leq \theta_{2}$. Thus, the connectivity is relatively independent of $P$ (match). This property of the phenomenological model is simply due to our assumption that a clone's connectivity has to be in the right range for it to be maintained in the network.

We have tested the robustness of these results by changing the bell-shaped function eqn (7) into the piecewise linear function

$$
f(C)= \begin{cases}C / \theta & \text { if } C \leq \theta \\ (2 \theta-C) / \theta & \text { if } \theta<C<2 \theta \\ 0 & \text { if } C \geq 2 \theta\end{cases}
$$

that is displayed in Fig. 14(a). Similar functions have been proposed before (Weisbuch et al., 1990). At the connectivity $C=\theta$ clones have an infinite lifetime. For consistency with the parameter choices made with the bell-shaped model, we have set $\theta=10$. Solving for $\bar{n}$ as a function of $P$ (match) we obtained very similar results to those displayed in Fig. 13(c) and (d). Thus, the existence of multiple equilibria in the phenomenological model does not depend on the sigmoid shape of the bell-shaped function [i.e. eqn (7)]. We found a difference however in the relation between the suppressive equilibrium values of $\bar{n}$ and $M$ [i.e. the relation depicted in Fig. 13(b)]. Instead of being linear, $\bar{n}$ grows proportionally to $\sqrt{M}$ in the piecewise linear model. Solving for the suppressive equilibrium of the eqn (8) boils down to substituting $f(C)=(2 \theta-C) / \theta$ into eqn (8) and solving a quadratic equation. For $\boldsymbol{M} \rightarrow \infty$ the corresponding solution is proportional to $\sqrt{\boldsymbol{M}}$. In Fig. 14(b) we plot the same simulation data as were shown in Fig. 11(a) fitted-using a least squares algorithm - to the two different relationships predicted by the phenomenological model. The light line is the best linear fit predicted by bell-shaped function: $\bar{n}=88 \cdot 2+1 \cdot 14 M, r^{2}=0 \cdot 78$. The heavy line is the best-fit predicted by the 

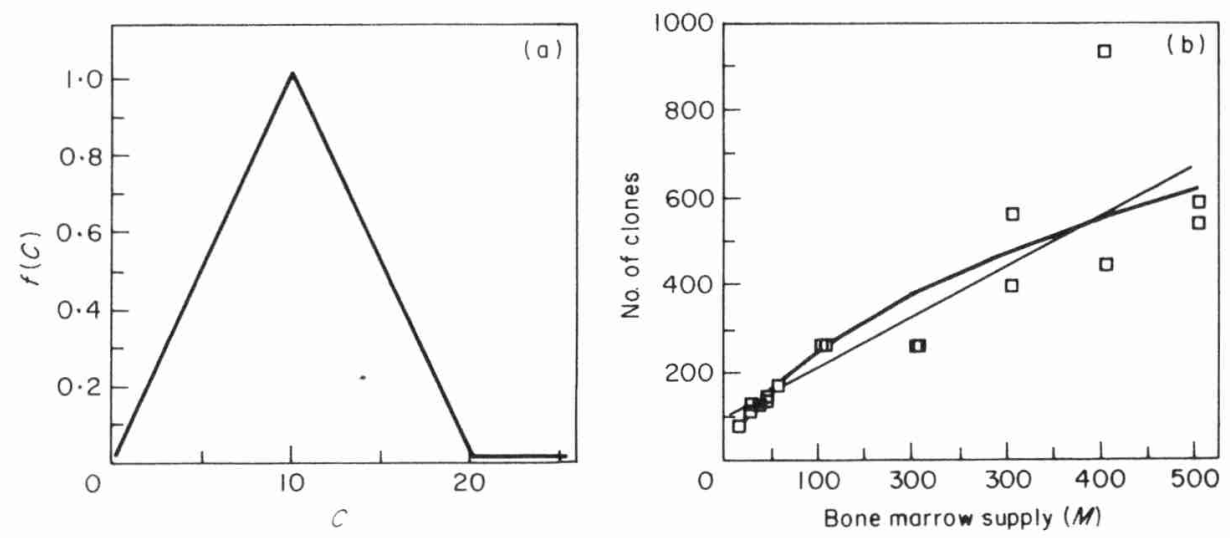

FIG. 14. The piece-wise linear function $f(C)$. (a) $f(C)$ as a function of the connectivity $C$. The maximum value of $f(C)$ is reached at $C=\theta=10$. (b) The simulation data of Fig. 11(a) ( $\square$ ) fitted as a linear function of $M$ (the light line) and as a function of $\sqrt{M}$ (the heavy line).

piece-wise linear function: $n=-45 \cdot 7+29 \cdot 2 \sqrt{M}, r^{2}=0 \cdot 78$. Since both fits are very similar within the range of $M$ values that we are able to study with the simulation model, we think this difference not very conclusive. It is clear, however, that the relation between $\bar{n}$ and $M$ depends on the particular choice of $f(C)$.

In conclusion, several properties of our phenomenological model, eqns ( 7 and $8)$, have corresponding features in the simulation model: (1) the existence of multiple and qualitatively different equilibria, (2) the inverse relation between $\bar{n}$ and $P$ (match), (3) the linear relation between $\bar{n}$ and $M$ (or $\sqrt{M}$ ), and (4) the constant connectivity. Thus, our interpretation of the behavior of the networks goes beyond the simple filling of shape space. Rather, it involves the rate of turnover of clones as a function of their connectivity in the system. This explains why the connectivity and the average field are such constant properties of the networks.

\section{Immunological Interpretations}

Throughout this paper we have refrained from discussing immune responses to antigens. Instead, we focussed on the development of the immune network in isolation from self and non-self antigens. As explained in our introduction, Coutinho (1989) and Holmberg et al. (1989) suggested that $10-20 \%$ of clones form a network and are not involved in immune responses to antigen; the other $80-90 \%$ of the clones remain disconnected (functionally at least) from the network and form the clonal part of the system. These disconnected clones remain in the resting state until they are activated by antigen, and then respond to generate the typical immune response measured by immunologists. Here we will touch upon some of the immunological implications of our results. These issues will be addressed more rigorously in a future publication. 
The fact that the observed equilibrium sizes of the networks are generally larger than the $n_{99}$ values [see Figs 7(a), 9(a), and 10(a)] suggest that the coverage of shape space is nearly complete. This means that, irrespective of the specificity of receptors [i.e. of $P$ (match)], any random bit pattern is expected to be seen by the network. We call this property the "self-regulatory completeness" of the repertoire: the higher the specificity of the receptors the larger the number of clones in the immune network automatically becomes so that the repertoire remains complete.

\section{CLONAL ORGANIZATION}

Recall that in our simulations we ignored those clones that failed to expand following introduction into the system. Since the basic mode of the network is suppression-typically $60 \%$ of the clones have suppressive fields-most of the ignored clones are expected to have a suppressive field. Checking the fields of the ignored clones we confirmed this expectation (not shown): most of these clones are ignored because they are suppressed. Immunologically, the suppressed clones are not of interest because they would be unresponsive to further stimulation by antigen. For low values of $P$ (match) and low values of $M$ however, e.g. $T=10,11$, $P($ match $)=0.005,0.012, M=10,20,40$ a significant proportion of the ignored clones failed to expand due to a lack of stimulation. This proportion varies around $25 \%$ of all ignored clones (not shown). These clones are functionally disconnected from the network: due to the completeness they are recognized by other clones but the degree of stimulation is low. Since they are perfectly capable of responding to further stimulation by antigens they form a "functionally disconnected" or "clonal" component of the immune system.

Thus, Coutinho's hypothesis that the immune system combines a highly connected network with a disconnected clonal system seems to be supported by these results. The results obtained with the phenomenological model, eqns ( 7 and 8), explain why such clones only occur when $M$ and $P$ (match) are small. Functionally disconnected clones should prevail in the equilibria dominated by poor stimulation. In an admittedly very speculative way, we may attempt to scale these observations to biologically reasonable values of $M$ and $P$ (match) by assuming that our phenomenological model remains valid in these regions. Figure 13(d) shows that for any value of $M$, a $P$ (match) can be found in which the stimulatory regime exists. Here a network can coexist with disconnected clones. In conclusion, Coutinho's hypothesis would be feasible if immune networks were to reside in this stimulatory regime.

\section{SUPPRESSION}

When B cells from a normal mouse are grown in culture with the B cell mitogen lipopolysaccharide (LPS), only 33\% of the cells grow (Andersson et al., 1977). One possible explanation for this as yet unexplained observation is that the majority of B cells are already suppressed by network interactions. Within the context of our 
model LPS would need to act in such a way as to increase the field of a cell. There is some evidence that LPS clusters immunoglobulin receptors (J. Andersson, personal communication). If this is in fact the case, then we would expect LPS to increase the field of a B cell and to have little effect on cells already suppressed by antiidiotype. In any event, our model supports the suggestion of Jerne (1974) that most clones are suppressed by network interactions.

\section{SELF TOLERANCE}

How the immune system discriminates between foreign antigens-to which it responds-and self antigens-to which it should not respond-is one of the most important unresolved problems in immunology. In fact, the whole idea of an autonomous network involving a core of $10-20 \%$ of all lymphocytes is intimately related to the issue of the self antigens (Pereira et al., 1986; Huetz et al., 1988; Coutinho, 1989; Holmberg et al., 1989). Empirical data show that the multireactive IgM antibodies, which comprise the early core of these networks, are often reactive to self antigens. The autonomous activity of these networks may therefore well be initiated by self antigens. Thus, it is argued that self-reactivity is physiological (Cohen, 1986; Coutinho, 1989). If clones reacting with self antigens always connect to the network, one can argue that the network contributes to self non-self discrimination because self reactive clones are deleted from the disconnected set of immunocompetent clones (Coutinho, 1989).

We have tested this idea by incorporating self antigens in the model. Antigens were incorporated as bit-patterns similar to the idiotypes. This increases the field of all self-reactive clones. In our model, the presence of self antigens in sufficiently high concentrations will suppress all clones that recognize them. However, self antigens that are present in stimulatory concentrations always gave rise to unlimited self-aggression. The network is capable of controlling some of the self-reactive clones by "over-stimulation"; see also De Boer (1989). This is particularly true for clones with many idiotypic connections (not shown). Clones with only few connections however, easily escape from network mediated control of autoreactivity. These clones expand because they are stimulated by the self antigen(s). When the selfreactive clones finally attain a large population size, they suppress their idiotypic partners in the network, thus freeing themselves further from network control (cf. De Boer \& Hogeweg, 1989a).

Thus, although the network's tendency to select for specificity seems suggestive in terms of self non-self discrimination, our B cell network fails to account for self non-self discrimination at all concentrations of self antigen. This need not be a shortcoming of our B cell network because self non-self discrimination probably takes place at the level of $\mathrm{T}$ cells. In the absence of $\mathrm{T}$ cell help, self antigens fail to activate $B$ cells and self non-self discrimination will occur.

\section{MEMORY}

Because we study a developing B cell network during early ontogeny, and because we have ignored $\mathrm{T}$ cells, memory need not be a property of our model. We 
nevertheless tested our network for its memory properties by temporarily introducing an antigen into a network that had attained an equilibrium size. Antigen is provided in the form of a random bit-string providing a constant stimulatory field. We observed two transient forms of memory, which will be described in detail elsewhere. First, we found that clones that would otherwise fail to be incorporated into the network due to a lack of stimulation, can now be stimulated by the antigen, and be maintained in the system because they are incorporated into the network. This would account for a form of idiotypic memory (Coutinho, 1989). Second, we observed that following the introduction and the removal of antigen, the network increased the incorporation of clones recognizing the antigen. Apparently, the antigen perturbed regions of the shape space causing those regions to provide a stimulatory signal to the clones recognizing the antigen. In other words, the network enlarged its repertoire of antigen reactive clones. Such a form of memory, in which novel gene combinations turn up in the repertoire following antigenic stimulation, has empirically been described as a "repertoire shift" (Berek et al., 1985; Berek \& Milstein, 1988).

\section{Discussion}

We have derived the following main conclusions for our model network:

- The equilibrium size of the idiotypic network is a property that emerges from interactions among the clones in a network. It is inversely related to the matching probability of immunoglobulin receptors. Networks comprised of sticky, or multireactive, receptors are small and typically contain hundreds of clones, whereas networks comprised of specific receptors are much larger. This dependence of size on specificity accounts for a form of "self-regulatory completeness". Networks grow large enough so that their repertoires are complete.

- Once a network is established it selects an immune repertoire. The number of clones in the network, and the number of disconnected immunocompetent clones, are much smaller than the total number of clones supplied by the bone marrow. The clones that survive interact with the network and thus the network influences the immune repertoire. Additionally, the network influences the repertoire of the clones remaining functionally disconnected from the network.

- The connectivity of the network, defined as the average number of connections per clone, is another emergent property and is relatively independent of the matching probability of the receptors.

- The number of different clones in the network and their connectivity are very large during the first month, and then decrease later in life. The initial peak in the number of clones is due to the fact that each clone is small and thus the system has not yet filled with antibody.

- Constant connectivity is achieved in the network by a selection process that determines the number of clones to be incorporated into the network.

- Networks select for clones with few connections. Specific clones are bound to win the competition for membership in the network, since multireactive clones 
might express multireactive receptors while later ones might use more specific receptors. Our phenomenological model suggests that if $P$ (match) were to decrease during ontogeny, the network will at some point in time switch from a suppressive equilibrium to a stimulatory equilibrium. From then on the combination of a network with functionally disconnected clones becomes feasible. In a previous study we modeled the change in receptor specificity by altering the binding threshold $T$ during ontogeny (De Boer, 1989). Alternatives would be to change the requirements for activation during ontogeny (i.e. to change $\theta_{1}$ or $\theta_{2}$ ), and/or to incorporate T-B cell co-operation. Empirical evidence suggests that $\mathrm{T}$ cells appear somewhat later in ontogeny, and that T and B cell repertoires influence each other (Huetz et al., 1988; Martinez-A et al., 1988). The model can be extended with T-B cell co-operation along the lines suggested by De Boer \& Hogeweg (1989c) and Farmer (1990).

Our present results are in agreement with the counterintuitive hypothesis (Coutinho, 1989) that the immune system combines a highly connected network with a functionally disconnected clonal system. Such a combination would reconcile the longstanding controversy between the two main views of the immune system, namely that of a clonal and a network organization.

Work performed under the auspices of the U.S. Department of Energy, and supported in part by NIH Grants AI 28433, RR 06555, and by the Sante Fe Institute through their Theoretical Immunology Program. An earlier version of this manuscript was discussed in great detail with Dr Gérard Weisbuch during the ISI meeting "Complexity and Evolution" held in Turin, 1990. We thank him for suggesting the shape space analysis. We thank Dr Geoffrey Hoffmann for criticizing an earlier version of the manuscript.

\section{REFERENCES}

ADAM, G. (1978). Theoretical models of lymphocyte network interactions in ontogenetic generation of antibody diversity. In: Theoretical Immunology (Bell, G. I., Perelson, A. S. \& Pimbley, G. H. Jr., eds) pp. 603-627. New York: Marcel Dekker.

Andersson, J., Coutinho, A., Lernhardt, W. \& Melchers, F. (1977). Clonal growth and maturation to immunologlobulin secretion in vitro of every growth inducible B lymphocyte. Cell 10, 27-34.

Bagley, R. J., Farmer, J. D., Packard, N. H., Perelson, A. S. \& Stadnyk, I. M. (1989). Modeling adaptive biological systems. BioSystems 23, 113-138.

Berek, C., Griffiths, G. M. \& Milstein, C. (1985). Molecular events during maturation of the immune response to oxazolone. Nature, Lond. 316, 314-318.

Berek, C. \& Milstein, C. (1988). The dynamic nature of the antibody repertoire. Immunol. Rev. 105, $5-26$.

Bona, C. A. (1988). V genes encoding autoantibodies molecular and phenotypic characteristics. Ann. Rev. Immunol. 6, 327-358.

Cohen, I. R. (1986). Regulation of autoimmune disease physiological and therapeutic. Immunol. Rev. 94, 5-21.

COSEnza, H. \& Köhler, H. (1972). Specific suppression of the antibody response by antibodies to receptors. Proc. natn. Acad. Sci. U.S.A. 69, 2701-2705.

Coutinho, A. (1989). Beyond clonal selection and network. Immunol. Rev. 110, 63-87.

Coutinho, A., Forni, L., Holmberg, Ivars, F. \& VAZ, N. N. (1984). From an antigen centered, clonal perspective of immune responses to an organism centered, network perspective of autonomous activity in a self-referential system. Immunol. Rev. 79, 151-169.

Coutinho, A., Marquez, C., Araujo, P. M. F., Pereira, P., Toribio, M. L., Marcos, M. A. R. \& MARTINEZ-A, C. (1987). A functional idiotypic network of T helper cells and antibodies, limited to the compartment of "naturally" activated lymphocytes in normal mice. Eur. J. Immunol. 17, 821-825. 
Darnell, J. E., Lodish, H. \& Baltimore, D. (1986). Molecular Cell Biology, pp. 1108-1109, New York: Scientific American Books.

DE BOER, R. J. (1983). GRIND: Great Integrator Differential Equations The Netherlands: Bioinformatics Group, University of Utrecht.

DE BOER, R. J. (1988). Symmetric idiotypic networks: connectance and switching, stability, and suppression. In: Theoretical Immunology Part 2 (Perelson, A. S., ed.) pp. 265-289. SFI Studies in the Science of Complexity Vol. III. City, CA: Addison-Wesley, Redwood.

DE BOER, R. J. (1989). Poor repertoire selection in symmetric idiotypic network models. Immunol. Lett. 22, 101-112.

De Boer, R. J. \& Hogeweg, P. (1989a). Memory but no suppression in low-dimensional symmetric idiotypic networks. Bull. math. Biol. 51, 223-246.

De Boer, R. J. \& Hogeweg, P. (1989b). Unreasonable implications of reasonable idiotypic network assumptions. Bull. math. Biol. 51, 381-408.

DE BOER, R. J. \& HoGEwEG, P. (1989c). Idiotypic network models incorporating T-B cell cooperation: the conditions for percolation. J. theor. Biol. 139, 17-38.

De Boer, R. J., Kevrekidis, I. G. \& Perelson, A. S. (1990). A simple idiotypic network model with complex dynamics. Chem. Engng. Sci. 45, 2375-2382.

Dibrov, B. F., Livshits, M. A. \& Volkenstein, M. V. (1977). Mathematical model of immune responses. J. theor. Biol. 65, 609-631.

DOEDEL, E. J. (1981). AUTO: a program for the bifurcation analysis of autonomous systems. Cong. Num. 30, 265-285.

Farmer, J. D. (1990). A Rosetta stone for connectionism. Physica D42, 153-187.

Farmer, J. D., Kauffman, S. A., Packard, N. H. \& Perelson, A. S. (1987). Adaptive dynamic networks as models for the immune system and autocatalytic sets. Ann. N. Y. Acad. Sci. 504, 118-130.

Farmer, J. D., Packard, N. H. \& Perelson, A. S. (1986). The immune system, adaptation and machine learning. Physica 22D, 187-204

FELler, W. (1968). An Introduction to Probability Theory and its Applications Vol. 1, 3rd edn, pp. 323-326, New York: Wiley.

FOWLER, A. C. (1981). Approximate solution of a model of biological immune responses incorporating delay. J. math. Biol. 13, 23-45.

GOLDBERG, R. J. (1952). A theory of antibody-antigen interactions. I. Theory for reactions of multivalent antigens with bivalent and univalent antibody. J. Am. Chem. Soc. 74, 5715-5725.

Gunther, N. \& HoffmanN, G. W. (1982). Qualitative dynamics of a network model of regulation of the immune system; a rationale for the IgM to IgG switch. J. theor. Biol. 94, 815-855.

HARDY, R. R. \& HAYAKAWA, K. (1986). Development and physiology of Ly-1 B and its human homolog, LEU-1 B. Immunol. Rev. 93, 53-79.

Herzenberg, L. A., Stall, A. M., Lalor, P. A., Sidman, C., Moore, W. A., Sparks, D. R. \& Herzenberg, L. A. (1986). The Ly-1 B cell lineage. Immunol. Rev. 93, 81-102.

Hiernaux, J., Bona, C. \& Baker, P. (1981). Neonatal treatment with low doses of antiidiotypic antibody leads to the expression of a silent clone. J. exp. Med. 153, 1004-1008.

HOFFMANN, G. W. (1975). A theory of regulation and self-nonself discrimination in an immune network. Eur. J. Immunol. 5, 638-647.

Hoffmann, G. W., Kion, T. A., Forsyth, R. B., Soga, K. G. \& Cooper-Willis, A. (1988). The N-dimensional network. In: Theoretical Immunology Part 2 (Perelson, A. S., ed.) pp. 291-319. SFI Studies in the Science of Complexity Vol. III Redwood City, CA: Addison-Wesley.

Holmberg, D., Anderson, A., Carlson, L. \& Forsgen, S. (1989). Establishment and functional implications of B-cell connectivity. Immunol. Rev. 110, 89-103.

holmberg, D., Freitas, A. A., Portnoï, D., Jacquemart, F. \& Coutinho, A. (1986a). Antibody repertoires of normal BALB/c mice: B lymphocyte populations defined by state of activation. Immunol. Rev. 93, 147-169.

Holmberg, D., Forsgen, S., Ivars, F. \& Coutinho, A. (1984). Reactions among IgM antibodies derived from normal neonatal mice. Eur. J. Immunol. 14, 435-441.

Holmberg, D., Wennerstrom, G., Anrade, L. \& Coutinho, A. (1986b). The high idiotypic connectivity of "natural" newborn antibodies is not found in the adult mitogen-reactive B cell repertoires. Eur. J. Immunol. 16, 82-87.

hooykaas, H., Benner, R., Pleasants, J. R. \& Wostmann, B. S. (1984). Isotypes and specificities of immunoglobulins produced by germ-free mice fed chemically defined "antigen-free" diet. Eur. $J$. Immunol. 14, 1127-1130.

huetz, F., Jacquemart, F., Peña Rossi, C., Varela, F. \& Coutinho, A. (1988). Autoimmunity: the moving boundaries between physiology and pathology. J. Autoimmun. 1, 507-518. 
Huetz, F., Sciard-larsson, E. L., Pereira, P., Portnoï, D. \& Coutinho, A. (1988). T Cell dependence of the "natural" autoreactive B cell activation in the spleen of normal mice. Eur. J. Immunol. 18, 1615-1622.

Hsu, D.-H., Sercarz, E. E. \& Miller, A. (1989). Internal connectivity is pervasive among primary and secondary anti-hen egg white lysozyme (HEL) IgG monoclonal antibodies. Internat. Immunol. 1, 197-204.

JERNE, N. K. (1974). Towards a network theory of the immune system. Ann. Immunol. (Inst. Pasteur) 125, 373-389.

KANG, C.-Y. \& KöHLER, H. (1986). A novel chimeric antibody with circular network characteristics: autobody. Ann. N.Y. Acad. Sci. 475, 114-122.

KAUFFMAN, S. A. (1969). Metabolic stability and epigenesis in randomly constructed genetic nets. $J$. theor. Biol. 22, 437-467.

KAVERI, S.-V., HAlpern, R., KANG, C.-Y.\& KöHler, H. (1990). Self-binding antibodies (autobodies) form specific complexes in solution. J. Immunol. 145, 2533-2538.

KEARNEY, J. F. \& VAKIL, M. (1986). Idiotype-directed interactions during ontogeny play a major role in the establishment of the adult B cell repertoire. Immunol. Rev. 94, 39-50.

KeARNeY, J. F., VAKIL, M. \& NiCholson, N. (1987). Non-random $\mathrm{V}_{\mathrm{H}}$ gene expression and idiotype anti-idiotype expression in early B cells. In: Evolution and Vertebrate immunity: the antigen Receptor and MHC Gene Families (Kelsoe, G. \& Schulze, D., eds) pp. 373-389. Austin: University of Texas Press.

Kesloe, G. \& CERnY, J. (1979). Reciprocal expansions of idiotypic and anti-idiotypic clones following antigen stimulation. Nature, Lond 279, 333-334.

LANGTON, C. (1990). Computation at the edge of chaos: phase transitions and emergent computation. Physica D42, 12-37.

Lorenz, E. N. (1963). Deterministic nonperiodic flow. J. Atmos. Sci. 20, 130-141.

Lundkvist, I., Coutinho, A., Varela, F. \& Holmberg, D. (1989). Evidence for a functional idiotypic network amongst natural antibodies in normal mice. Proc. natn. Acad. Sci. U.S.A. 86, 5074-5078.

Martinez-A, C., Pereira, P., Toribo, M. L., Marcos, M. A. R., Bandeira, A., De la Hera, A., Marquez, C., Cazenave, P.-A. \& Coutinho, A. (1988). The participation of B cells and antibodies in the selection and maintenance of T cell repertoires. Immunol. Rev. 101, 191-215.

Masmoudi, H., Mota-Santos, T., Huetz, F., Coutinho, A. \& Cazenave, P.-A. (1990). All T15 Id-positive antibodies (but not the majority of $\mathrm{V}_{\mathrm{H}} \mathrm{T} 15^{+}$antibodies) are produced by peritoneal $\mathrm{CD} 5^{+}$ B lymphocytes. Internat. Immunol. 2, 515-520.

Opstelten, D. \& Osmond, D. G. (1983). Pre-B cells in mouse bone marrow: immunoflorescence stathmokinetic studies of the proliferation of cytoplasmaic $\mu$-chain-bearing in normal mice. J. Immunol. $131,2635-2640$.

PACKARD, N. H. (1988). Adaptation toward the edge of chaos. In: Dynamic Patterns in Complex Systems (Kelso, J. A. S., Mandell, A. J. \& Shlesinger, M. F., eds) Singapore: World Scientific.

Pereira, P., Forni, L., Larsson, E. L., Cooper, M., Heusser, C. \& Coutinho, A. (1986). Autonomous activation of $\mathrm{B}$ and $\mathrm{T}$ cells in antigen-free mice. Eur. J. Immunol. 16, 685-688.

PERELSON, A. S. (1984). Some mathematical models of receptor clustering by multivalent ligands. In: Cell Surface Dynamics: Concepts and Models (Perelson, A. S., DeLisi, C. \& Wiegel, F. W., eds) pp. 223-276. New York: Marcel Dekker.

PERElson, A. S. (1988). Towards a realistic model of the immune network. In: Theoretical Immunology Part Two (Perelson, A. S., ed.) pp. 377-401 SFI Studies in the Science of Complexity Vol. III. Redwood City, CA: Addison-Wesley

Perelson, A. S. (1989a). Immune network theory. Immunol. Rev. 110, 5-36.

Perelson, A. S. (1989b). Immune networks: a topological view. In: Cell to Cell Signalling: From Experiments to Theoretical Models (Goldbeter, A., ed.) pp. 261-262. London: Academic Press.

Perelson, A. S. \& Delisi, C. (1980). Receptor clustering on a cell surface. I. Theory of receptor cross-linking by ligands bearing two chemically identical functional groups. Math. Biosci. 48, 71-110.

Post, W. M. \& PiMm, S. L. (1983). Community assembly and food web stability. Math. Biosci. 64, 169-192.

Press, W. H. Flannery, B. P., Teukolsky, S. A. \& Vetterling, W. T. (1988). Numerical Recipes in C. the Art of Scientific Computing. Cambridge: Cambridge University Press.

Ric HTER, P. H. (1975). A network theory of the immune system. Eur. J. Immunol. 5, 350-354.

RICHTER, P. H. (1978). The network idea and the immune response. In: Theoretical Immunology (Bell, G. I., Perelson, A. S. \& Pimbley, G. H., eds) pp. 539-569. New York: Marcel Dekker.

ROIJKEY, L. S. \& ADLER, F. L. (1983). Regulation of natural antiallotype antibody responses by idiotypic network-induced auto-antiidiotypic antibodies. J. exp. Med. 157, 1920-1931. 
Segel, L. A. \& Perelson, A. S. (1988). Computations in shape space: a new approach to immune network theory. In: Theoretical Immunology Part 2 (Perelson, A. S., ed.) pp. 321-343 SFI Studies in the Science of Complexity Vol. III. Redwood City, CA: Addison-Wesley.

Segel, L. A. \& Perelson, A. S. (1989). Shape space analysis of immune networks. In: Cell to .Cell Signalling: From Experiments to Theoretical Models (Goldbeter, A., ed.) pp. 273-283. New York: Academic Press.

SpARrow, C. (1982). The Lorenz Equations: Bifurcations, Chaos, and Strange Attractors. New York: Springer.

StewART, J. \& VARELA, F. J. (1989). Exploring the meaning of connectivity in the immune network. Immunol. Rev. 110, 37-61.

Stewart, J. \& VAREla, F. J. (1990). Dynamics of a class of immune networks. II. Oscillatory activity of cellular and humoral components. J. theor. Biol. 144, 103-115.

STOCKMEYER, W. H. (1943). Theory of molecular size distributions and gel formation in branched-chain polymers. J. chem. Phys. 11, 45-55.

Vakil, M., Sauter, H., Paige, C. \& Kearney, J. F. (1986). In vivo suppression of perinatal multispecific B cells results in a distortion of the adult B cell repertoire. Eur. J. Immunol. 16, 1159-1165.

Varela, F. J., Coutinho, A., Dupire, B. \& VAz, N. N. (1988). Cognitive networks: immune, neural, and otherwise. In: Theoretical Immunology Part Two (Perelson, A. S., ed.) pp. 359-375 SFI Studies in the Science of Complexity Vol. III. Redwood City CA: Addison-Wesley.

VAREla, F. J. \& STEWART, J. (1990). Dynamics of a class of immune networks. I. Global stability of idiotype interactions. $J$. theor. Biol. 144, 93-101.

VAZ, N. N. \& VARELA, F. J. (1978). Self and non-sense: an organism-centered approach to immunology. Med. Hypothesis 4, 231-267.

WEINAND, R. G. (1990). Somatic mutation, affinity maturation and the antibody repertoire: a computer model. J. theor. Biol. 143, 343-382.

WEISBUCH, G. (1989). Dynamical behavior of discrete models of Jerne's network. In: Theories of Immune Networks (Atan, H. \& Cohen, I. R., eds) pp. 53-62, Springer Series in Synergetics, Berlin: Springer.

Weisbuch, G., De Boer, R. J. \& Perelson, A. S. (1990). Localized memories in idiotypic networks. J. Theor. Biol. 146, 483-499.

\section{APPENDIX}

\section{Estimating the parameter $\boldsymbol{d}_{C}$}

To obtain a realistic range of $d_{C}$ values, note that the parameter $d_{C}$ appears only in the term $-d_{C} \sum \mathbf{J}_{i j} A_{i} A_{j}$. Assuming that free antibodies and complexes rapidly come to equilibrium, the equilibrium concentration of complexes is proportional to $\sum \mathbf{J}_{i j} A_{i} A_{j}$, and hence $d_{C}$ is proportional to the rate of complex removal. We shall assume that the time for complex removal by macrophages and other scavenger cells is of order $0 \cdot 1$ days. To obtain a precise value of $d_{C}$ we need to analyze the full term $-d_{C} \sum \mathbf{J}_{i j} A_{i} A_{j}$ in more detail. In this paper we have scaled affinities so that $\mathbf{J}_{i j}=1$. If realistic affinity values were used, of order of magnitude $K$, then the field $h$ would be multiplied by $K$. Dividing the thresholds $\theta_{1}$ and $\theta_{2}$ by $K$ would leave the activation function $f\left(\bar{A}_{i}\right)$ invariant. Thus, this choice of $\mathbf{J}_{i j}=1$ does not affect $f\left(\bar{A}_{i}\right)$ as long as $\theta_{1}$ and $\theta_{2}$ are chosen appropriately. Similarly, multiplying $\mathbf{J}_{i j}$ by $K$ would affect the concentration of complexes. Thus, we shall choose $d_{C}$ to reflect the incorporation of typical affinity values.

In early immune networks the antibodies found in the serum that have high connectivity and that are thought to play an important role are the natural IgM antibodies. The equilibrium concentration of complexes composed of IgM antibodies is of order $100 K A_{i} A_{j}$, where the factor of 100 is a statistical factor indicating that any of the ten binding sites on one $\operatorname{IgM}$ can interact with any of the ten binding sites on the other $\operatorname{IgM}$, and $K$ is the usual single-site binding affinity. Realistic 
values of affinity are $K=10^{4}-10^{7} \mathrm{M}^{-1}$. In this paper we have chosen to express antibody concentrations in "units" per animal rather than moles/liter. Here a unit is the amount of antibody secreted by a maximally stimulated B cell in 1 day. Using a maximal secretion rate of $10^{4}$ molecules $\mathrm{sec}^{-1}$, one unit corresponds to approximately $10^{-15}$ moles. Our simulation results are compared with data obtained in mice, and thus we shall assume that the appropriate volume, is the circulatory system volume of a mouse, approximately $1 \mathrm{~cm}^{3}$. Hence 1 unit $=10^{-12} \mathrm{M}$. Finally, $d_{C}$ is $10 \mathrm{day}^{-1}$ (the rate of complex removal), multiplied by $100 \mathrm{~K}$, and hence $d_{C}=10^{3}$ $K=10^{7}-10^{10}$ day $^{-1} \mathrm{M}^{-1}=10^{-5}-10^{-2}$ day $^{-1}$ units $^{-1}$. Because our model only follows the creation of $1: 1$ idiotype anti-idiotype complexes and neglects the formation of larger complexes, which can also occur and lead to antibody depletion, we compensate for this neglect by choosing values of $d_{C}$ at the high end of the range. We shall thus use $d_{C}=10^{-3}$ as a typical value, and explore the effects of choosing $d_{C}=10^{-2}$. 